\title{
Optimal Control of Nonlinear Switched Systems: Computational Methods and Applications
}

\author{
Qun Lin · Ryan Loxton · Kok Lay Teo
}

Received: 16 June 2013 / Revised: 18 July 2013 / Accepted: 22 July 2013 /

Published online: 6 September 2013

(C) Operations Research Society of China, Periodicals Agency of Shanghai University, and

Springer-Verlag Berlin Heidelberg 2013

\begin{abstract}
A switched system is a dynamic system that operates by switching between different subsystems or modes. Such systems exhibit both continuous and discrete characteristics - a dual nature that makes designing effective control policies a challenging task. The purpose of this paper is to review some of the latest computational techniques for generating optimal control laws for switched systems with nonlinear dynamics and continuous inequality constraints. We discuss computational strategies for optimizing both the times at which a switched system switches from one mode to another (the so-called switching times) and the sequence in which a switched system operates its various possible modes (the so-called switching sequence). These strategies involve novel combinations of the control parameterization method, the timescaling transformation, and bilevel programming and binary relaxation techniques. We conclude the paper by discussing a number of switched system optimal control models arising in practical applications.
\end{abstract}

Keywords Switched system $\cdot$ Nonlinear system $\cdot$ Optimal control $\cdot$ Nonlinear optimization

Mathematics Subject Classification 49M37 - 65K10 - 90C30 - 93C10

This work was supported by the Australian Research Council through Discovery Grant (No. DP110100083).

Q. Lin · R. Loxton · K.L. Teo $(\varangle)$

Department of Mathematics and Statistics, Curtin University, Perth, Australia

e-mail: k.1.teo@curtin.edu.au

Q. Lin

e-mail: q.lin@curtin.edu.au

R. Loxton

e-mail: r.loxton@curtin.edu.au 


\section{Introduction}

Many real-world systems operate by switching between different subsystems or modes. Such systems are called switched systems. An example of a switched system is a switched-capacitor DC/DC power converter, which operates by switching between different circuit topologies to produce a steady output voltage [18, 44]. Other examples of switched systems include robots [9], fed-batch bioreactors [35], DISC engines [52], and power management devices [51].

As with any system, switched systems are manipulated by varying certain input variables. These input variables could be time-varying signals (control signals) or time-invariant parameters (system parameters). The switching times (the times at which the switched system switches from one mode to another) and the mode sequence (the sequence in which the switched system operates its various possible modes) can also be considered as input variables. In this paper, we are concerned with switched system optimal control problems in which the input variables must be chosen judiciously so that some measure of system performance is maximized.

Switched system optimal control problems arise naturally when the control system under consideration has multiple operating modes. Such problems can also arise when solving conventional optimal control problems for single-mode systems. Indeed, by using the Pontryagin maximum principle [49], it is often possible to show that the optimal control for a conventional single-mode system is composed of a mixture of bang-bang and singular regimes, and these regimes can be considered as modes in an associated switched system model [56]. In this case, the conventional optimal control problem reduces to a switched system optimal control problem in which the aim is to determine the optimal switching times and/or optimal mode sequence.

The general switched system framework also encapsulates dynamic systems in which some (or all) of the control variables assume values in a discrete set. Such discrete-valued control variables arise in many applications, including submarine control [11], hybrid power systems [54], micro-robots [4], sensor scheduling [63], switching power amplifiers [57], subway trains [55], and gradient-elution chromatography [12]. An optimal control problem involving discrete-valued control variables is called an optimal discrete-valued control problem [27, 68]. Such problems can be viewed as switched system optimal control problems in which each discrete control value corresponds to a distinct mode. Thus, techniques for solving switched system optimal control problems are directly applicable to optimal discrete-valued control problems.

Optimal control theory for switched systems is now well developed; see [6, 10, 24, $58]$ and the references cited therein. However, for switched systems with nonlinear subsystems and/or nonlinear state constraints (which includes most switched systems arising in practice), analytical techniques are not sufficient to determine an optimal control policy. Thus, numerical optimization methods for switched systems are now attracting significant attention from the research community.

The control parameterization method [59] and the time-scaling transformation [27] are two fundamental numerical methods for solving nonlinear optimal control problems, including switched system optimal control problems. Control parameterization involves approximating the control by a linear combination of basis functions, 
where the coefficients in the linear combination are decision variables to be chosen optimally. The time-scaling transformation provides a mechanism for optimizing the knot points used in control parameterization, thereby increasing solution accuracy. The purpose of this paper is to review the latest advances in the control parameterization and time-scaling techniques for solving switched system optimal control problems.

The remainder of this paper is organized as follows. In Sect. 2, we introduce the mathematical formulation of a general switched system optimal control problem. In Sect. 3, we introduce the control parameterization method for the simple case in which both the switching times and the mode sequence are fixed and known. Then, in Sect. 4, we show how to combine the control parameterization method with the timescaling transformation to handle situations in which the switching times are decision variables to be optimized. In Sect. 5, we discuss three strategies for optimizing the mode sequence in a switched system. Finally, in Sect. 6, we describe some switched system optimal control models arising in practical applications.

\section{Switched System Optimal Control Problems}

We consider the following prototypical switched system with $m$ modes and $p$ stages:

$$
\begin{aligned}
\dot{\boldsymbol{x}}(t) & =\boldsymbol{f}^{v_{i}}(t, \boldsymbol{x}(t), \boldsymbol{u}(t), \zeta), \quad t \in\left[\tau_{i-1}, \tau_{i}\right), i=1, \cdots, p, \\
\boldsymbol{x}(0) & =\boldsymbol{x}^{0}
\end{aligned}
$$

where $t$ denotes time; $\boldsymbol{x}(t) \in \mathbb{R}^{n}$ is the state vector at time $t ; \boldsymbol{u}(t) \in \mathbb{R}^{r}$ is the control vector at time $t ; \zeta \in \mathbb{R}^{w}$ is the parameter vector; $\tau_{0}=0$ is the initial time; $\tau_{p}=T$ is the terminal time; $\tau_{i}, i=1, \cdots, p-1$, are the switching times or switching instants; $v_{i} \in\{1, \cdots, m\}$ is the active mode (one of $m$ possible modes) during the $i$ th stage (interval $\left.\left[\tau_{i-1}, \tau_{i}\right)\right) ; \boldsymbol{x}^{0} \in \mathbb{R}^{n}$ is a given initial state; and $\boldsymbol{f}^{j}: \mathbb{R} \times \mathbb{R}^{n} \times \mathbb{R}^{r} \times \mathbb{R}^{w} \rightarrow \mathbb{R}^{n}$, $j=1, \cdots, m$, are given continuously differentiable functions that define the subsystem dynamics.

System (2.1)-(2.2) operates as follows. It begins in state $\boldsymbol{x}^{0}$ at time $t=0$. From $t=0$ to $t=\tau_{1}$, the system runs smoothly according to (2.1) with $i=1$ (mode $v_{1}$ ). Then, at time $t=\tau_{1}$, the system switches from mode $v_{1}$ to mode $v_{2}$. After the mode switch, the system continues to run smoothly according to (2.1) with $i=2$ (mode $v_{2}$ ) until time $t=\tau_{2}$, at which point the system changes mode once again. The system continues to evolve in this manner until it reaches the terminal time $t=\tau_{p}=T$. The interval $[0, T]$ is called the time horizon.

In each mode, equation (2.1) expresses the rate of change of the system's state as a function of the current time, the current state, the current control, and the current parameter vector. The sequence of active modes $\left\{v_{1}, \cdots, v_{p}\right\}$ is called the mode sequence or switching sequence.

The control and parameter vectors in (2.1)-(2.2) are decision variables (or input variables) through which the system operator can influence the behavior of the system. The control vector is a time-varying decision variable and the parameter vector is a time-invariant decision variable. The aim is to manipulate these decision variables 
in an optimal manner so that system (2.1)-(2.2) performs as efficiently as possible. In many switched systems, the switching times and/or mode sequence are also decision variables to be chosen optimally. Thus, in general, there is no requirement that $p$ (the number of stages) is equal to $m$ (the number of possible modes).

As an example of a switched system, consider the following system of ordinary differential equations with discrete-valued control variables:

$$
\begin{aligned}
& \dot{\boldsymbol{x}}(t)=\boldsymbol{f}(t, \boldsymbol{x}(t), \boldsymbol{u}(t)), \quad t \in[0, T], \\
& \boldsymbol{x}(0)=\boldsymbol{x}^{0},
\end{aligned}
$$

where

$$
\boldsymbol{u}(t) \in\left\{\boldsymbol{u}^{1}, \cdots, \boldsymbol{u}^{m}\right\}, \quad t \in[0, T] .
$$

This system can be written in the form of (2.1)-(2.2) as follows:

$$
\begin{aligned}
\dot{\boldsymbol{x}}(t) & =\boldsymbol{f}\left(t, \boldsymbol{x}(t), \boldsymbol{u}^{v_{i}}\right), \quad t \in\left[\tau_{i-1}, \tau_{i}\right), i=1, \cdots, p, \\
\boldsymbol{x}(0) & =\boldsymbol{x}^{0}
\end{aligned}
$$

where $p-1$ is the number of control switches; $\tau_{i}, i=1, \cdots, p-1$, are the times at which the control switches from one discrete value to another; and $v_{i} \in\{1, \cdots, m\}$, $i=1, \cdots, p$, define the sequence in which the control assumes the various discrete values in $\left\{\boldsymbol{u}^{1}, \cdots, \boldsymbol{u}^{m}\right\}$. Thus, a system with discrete-valued control variables can be viewed as a switched system.

In some switched systems, the state vector experiences an instantaneous "jump" when the system switches from one mode to another. This so-called state jump is typically expressed as follows:

$$
\boldsymbol{x}\left(\tau_{i}^{+}\right)=\boldsymbol{\phi}^{i}\left(\boldsymbol{x}\left(\tau_{i}^{-}\right), \zeta\right), \quad i=1, \cdots, p-1,
$$

where $\boldsymbol{\phi}^{i}: \mathbb{R}^{n} \times \mathbb{R}^{w} \rightarrow \mathbb{R}^{n}, i=1, \cdots, p-1$, are given continuously differentiable functions and

$$
\boldsymbol{x}\left(\tau_{i}^{ \pm}\right)=\lim _{t \rightarrow \tau_{i} \pm} \boldsymbol{x}(t) .
$$

Switched systems with state jumps are called impulsive systems or switched impulsive systems in the literature. Note that the jump conditions (2.3) are still valid for switched systems without state jumps: we can simply define $\boldsymbol{\phi}^{i}=\boldsymbol{x}\left(\tau_{i}^{-}\right)$for each $i=1, \cdots, p-1$ so that no state jumps occur.

In any practical system, the control vector-an input to the system-cannot be unbounded. Thus, the components of the control vector must lie within specified lower and upper bounds:

$$
a_{l} \leqslant u_{l}(t) \leqslant b_{l}, \quad t \in[0, T], l=1, \cdots, r
$$

where $a_{l}$ and $b_{l}$ are given constants such that $a_{l}<b_{l}$. Any Borel measurable function $\boldsymbol{u}:[0, T] \rightarrow \mathbb{R}^{r}$ satisfying (2.4) is called an admissible control function. 
The components of the parameter vector are also bounded:

$$
c_{l} \leqslant \zeta_{l} \leqslant d_{l}, \quad l=1, \cdots, w,
$$

where $c_{l}$ and $d_{l}$ are given constants such that $c_{l}<d_{l}$. Any vector $\zeta \in \mathbb{R}^{w}$ satisfying (2.5) is called an admissible parameter vector.

In addition to the simple bound constraints (2.4) and (2.5), practical problems often contain more complicated constraints involving both the state and the control. We consider two types of mixed state-control constraints: canonical constraints and continuous inequality constraints.

Canonical constraints are expressed as follows:

$$
\Phi_{j}(\boldsymbol{x}(T), \zeta)+\int_{0}^{T} \mathcal{L}_{j}(t, \boldsymbol{x}(t), \boldsymbol{u}(t), \zeta) d t\left\{\begin{array}{l}
=0 \\
\geqslant 0
\end{array} \quad j \in \mathcal{J}_{1},\right.
$$

where $\mathcal{J}_{1}$ is a finite index set and $\Phi_{j}: \mathbb{R}^{n} \times \mathbb{R}^{w} \rightarrow \mathbb{R}$ and $\mathcal{L}_{j}: \mathbb{R} \times \mathbb{R}^{n} \times \mathbb{R}^{r} \times \mathbb{R}^{w} \rightarrow$ $\mathbb{R}, j \in \mathcal{J}_{1}$, are given continuously differentiable functions. The canonical form on the left-hand side of (2.6) encapsulates many of the common constraints arising in practice. For example, terminal state constraints in the form of $\boldsymbol{x}(T)=\boldsymbol{x}^{f}$, where $\boldsymbol{x}^{f}$ is a desired final state, can be modeled by a canonical equality constraint with $\Phi_{j}=\left\|\boldsymbol{x}(T)-\boldsymbol{x}^{f}\right\|^{2}$ and $\mathcal{L}_{j}=0$.

Each canonical function on the left-hand side of (2.6) evaluates to a single number, which must be either zero or non-negative, depending on whether the constraint is of equality or inequality type. Thus, each canonical constraint constitutes a single restriction on the system. Continuous inequality constraints, on the other hand, constitute an infinite number of restrictions: they are used to impose conditions that must be satisfied at every point in the time horizon. Such constraints are expressed as follows:

$$
h_{j}(t, \boldsymbol{x}(t), \boldsymbol{u}(t), \zeta) \geqslant 0, \quad t \in[0, T], j \in \mathcal{J}_{2},
$$

where $\mathcal{J}_{2}$ is a finite index set and $h_{j}: \mathbb{R} \times \mathbb{R}^{n} \times \mathbb{R}^{r} \times \mathbb{R}^{w} \rightarrow \mathbb{R}, j \in \mathcal{J}_{2}$, are given continuously differentiable functions.

In theory, a continuous inequality constraint in the form of (2.7) can be transformed into an equivalent canonical equality constraint with $\Phi_{j}=0$ and $\mathcal{L}_{j}=$ $\min \left\{h_{j}, 0\right\}^{2}$. However, this transformation is known to cause computational difficulties [45]. Thus, as explained later in this paper, a better approach is to use the constraint transcription method to approximate the continuous inequality constraints (2.7) by canonical inequality constraints.

To measure system cost, we define the following cost function:

$$
g_{0}=\Phi_{0}(\boldsymbol{x}(T), \zeta)+\int_{0}^{T} \mathcal{L}_{0}(t, \boldsymbol{x}(t), \boldsymbol{u}(t), \zeta) d t,
$$

where $\Phi_{0}: \mathbb{R}^{n} \times \mathbb{R}^{w} \rightarrow \mathbb{R}$ and $\mathcal{L}_{0}: \mathbb{R} \times \mathbb{R}^{n} \times \mathbb{R}^{r} \times \mathbb{R}^{w} \rightarrow \mathbb{R}$ are given continuously differentiable functions. Note that this cost function has the same form as the canonical constraints. 
Our switched system optimal control problem can now be stated as follows: Choose an admissible control function $\boldsymbol{u}$ and an admissible parameter vector $\zeta$ (as well as possibly the switching times $\tau_{i}, i=1, \cdots, p-1$, and the mode sequence $\left.\left\{v_{1}, \cdots, v_{p}\right\}\right)$ to minimize the cost function (2.8) subject to the switched system (2.1)(2.2), the jump conditions (2.3), the canonical constraints (2.6), and the continuous inequality constraints (2.7).

\section{Control Parameterization}

It is usually not possible to derive an analytical solution to a switched system optimal control problem. Thus, numerical methods are indispensable, especially for practical problems. One of the main hurdles with solving an optimal control problem numerically is that the value of the control vector needs to be determined at every point in the time horizon - an infinite number of points. To overcome this difficulty, control parameterization can be used to approximate the control signal by a piecewise-constant function. We now describe this approach as it pertains to switched systems.

\subsection{Control Approximation}

For simplicity, we assume that the switching times and mode sequence are fixed and known. This assumption is relaxed in subsequent sections.

Under mild conditions on the functions $f^{j}: \mathbb{R} \times \mathbb{R}^{n} \times \mathbb{R}^{r} \times \mathbb{R}^{w} \rightarrow \mathbb{R}^{n}, j=$ $1, \cdots, m$, it can be shown that the switched system (2.1)-(2.2) with jump conditions (2.3) admits a unique solution (see [1,3]). This solution is called the state trajectory. Let $\boldsymbol{x}(\cdot \mid \boldsymbol{u}, \boldsymbol{\zeta})$ denote the state trajectory corresponding to the admissible control $\boldsymbol{u}$ and the admissible parameter vector $\zeta$.

In the control parameterization method, each subinterval $\left[\tau_{i-1}, \tau_{i}\right)$ is decomposed into $q_{i}$ minor subintervals $\left[\gamma_{k-1}^{i}, \gamma_{k}^{i}\right), k=1, \cdots, q_{i}$, where $\gamma_{k}^{i}, k=0, \cdots, q_{i}$, are pre-fixed knot points such that

$$
\tau_{i-1}=\gamma_{0}^{i}<\gamma_{1}^{i}<\gamma_{2}^{i}<\cdots<\gamma_{q_{i}-1}^{i}<\gamma_{q_{i}}^{i}=\tau_{i} .
$$

The control function is then approximated by a constant vector on each minor subinterval:

$$
\boldsymbol{u}(t) \approx \boldsymbol{\sigma}^{i, k}, \quad t \in\left[\gamma_{k-1}^{i}, \gamma_{k}^{i}\right), k=1, \cdots, q_{i}, i=1, \cdots, p
$$

Let

$$
\boldsymbol{\sigma}^{i}=\left[\left(\sigma^{i, 1}\right)^{\top}, \cdots,\left(\sigma^{i, q_{i}}\right)^{\top}\right]^{\top}, \quad i=1, \cdots, p,
$$

and

$$
\boldsymbol{\sigma}=\left[\left(\boldsymbol{\sigma}^{1}\right)^{\top}, \cdots,\left(\boldsymbol{\sigma}^{p}\right)^{\top}\right]^{\top}
$$

The control approximation in (3.1) can be written as follows:

$$
\boldsymbol{u}(t) \approx \boldsymbol{u}^{q}(t \mid \boldsymbol{\sigma})=\sum_{i=1}^{p} \sum_{k=1}^{q_{i}} \boldsymbol{\sigma}^{i, k} \chi_{\left[\gamma_{k-1}^{i}, \gamma_{k}^{i}\right)}(t), \quad t \in[0, T),
$$




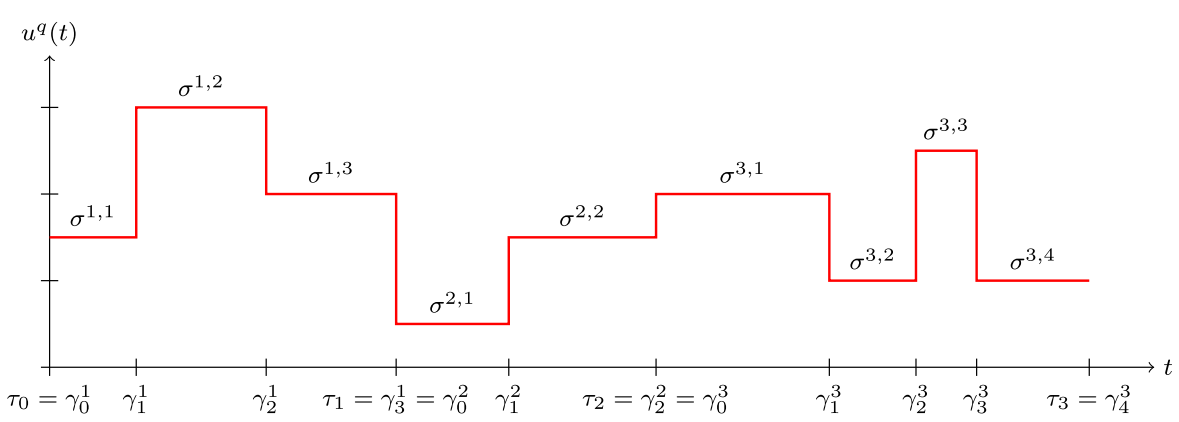

Fig. 1 A piecewise-constant control approximation with $p=3$ and $q=(3,2,4)$

where $q=\left(q_{1}, \cdots, q_{p}\right)$ and $\chi_{\left[\gamma_{k-1}^{i}, \gamma_{k}^{i}\right)}: \mathbb{R} \rightarrow \mathbb{R}$ is the indicator function defined by

$$
\chi_{\left[\gamma_{k-1}^{i}, \gamma_{k}^{i}\right)}(t)= \begin{cases}1, & \text { if } t \in\left[\gamma_{k-1}^{i}, \gamma_{k}^{i}\right), \\ 0, & \text { otherwise. }\end{cases}
$$

An example of $\boldsymbol{u}^{q}(\cdot \mid \boldsymbol{\sigma})$ is shown in Fig. 1. Note that (3.2) does not define the value of $\boldsymbol{u}^{q}(\cdot \mid \boldsymbol{\sigma})$ at the terminal time. By convention, we define $\boldsymbol{u}^{q}(T \mid \boldsymbol{\sigma})=\boldsymbol{u}^{q}\left(T^{-} \mid \boldsymbol{\sigma}\right)=$ $\boldsymbol{\sigma}^{p, q_{p}}$.

It follows from (2.4) that the components of $\sigma$ must satisfy the following bound constraints:

$$
a_{l} \leqslant \sigma_{l}^{i, k} \leqslant b_{l}, \quad l=1, \cdots, r, k=1, \cdots, q_{i}, i=1, \cdots, p .
$$

Substituting (3.2) into (2.1)-(2.3) gives

$$
\begin{aligned}
\dot{\boldsymbol{x}}(t) & =\boldsymbol{f}^{v_{i}}\left(t, \boldsymbol{x}(t), \boldsymbol{\sigma}^{i, k}, \zeta\right), \quad t \in\left[\gamma_{k-1}^{i}, \gamma_{k}^{i}\right), k=1, \cdots, q_{i}, i=1, \cdots, p, \\
\boldsymbol{x}\left(\tau_{i}^{+}\right) & =\boldsymbol{\phi}^{i}\left(\boldsymbol{x}\left(\tau_{i}^{-}\right), \zeta\right), \quad i=1, \cdots, p-1, \\
\boldsymbol{x}(0) & =\boldsymbol{x}^{0}
\end{aligned}
$$

Let $\boldsymbol{x}^{q}(\cdot \mid \boldsymbol{\sigma}, \boldsymbol{\zeta})$ denote the solution of (3.3)-(3.5) corresponding to the admissible pair $(\sigma, \zeta)$. Then clearly,

$$
\boldsymbol{x}^{q}(t \mid \boldsymbol{\sigma}, \zeta)=\boldsymbol{x}\left(t \mid \boldsymbol{u}^{q}(\cdot \mid \boldsymbol{\sigma}), \zeta\right), \quad t \in[0, T]
$$

Constraints (2.6) and (2.7) become

$$
\begin{aligned}
g_{j}^{q}(\boldsymbol{\sigma}, \boldsymbol{\zeta})= & \Phi_{j}\left(\boldsymbol{x}^{q}(T \mid \boldsymbol{\sigma}, \boldsymbol{\zeta}), \boldsymbol{\zeta}\right) \\
& +\sum_{i=1}^{p} \sum_{k=1}^{q_{i}} \int_{\gamma_{k-1}^{i}}^{\gamma_{k}^{i}} \mathcal{L}_{j}\left(t, \boldsymbol{x}^{q}(t \mid \boldsymbol{\sigma}, \boldsymbol{\zeta}), \boldsymbol{\sigma}^{i, k}, \boldsymbol{\zeta}\right) d t\left\{\begin{array}{l}
=0 \\
\geqslant 0
\end{array} \quad j \in \mathcal{J}_{1},\right.
\end{aligned}
$$


and

$h_{j}\left(t, \boldsymbol{x}^{q}(t \mid \boldsymbol{\sigma}, \zeta), \boldsymbol{\sigma}^{i, k}, \zeta\right) \geqslant 0, \quad t \in\left[\gamma_{k-1}^{i}, \gamma_{k}^{i}\right], k=1, \cdots, q_{i}, i=1, \cdots, p, j \in \mathcal{J}_{2}$.

Furthermore, the cost function (2.8) becomes

$$
g_{0}^{q}(\boldsymbol{\sigma}, \boldsymbol{\zeta})=\Phi_{0}\left(\boldsymbol{x}^{q}(T \mid \boldsymbol{\sigma}, \boldsymbol{\zeta}), \boldsymbol{\zeta}\right)+\sum_{i=1}^{p} \sum_{k=1}^{q_{i}} \int_{\gamma_{k-1}^{i}}^{\gamma_{k}^{i}} \mathcal{L}_{0}\left(t, \boldsymbol{x}^{q}(t \mid \boldsymbol{\sigma}, \boldsymbol{\zeta}), \boldsymbol{\sigma}^{i, k}, \boldsymbol{\zeta}\right) d t
$$

Thus, after applying the control parameterization method, the switched system optimal control problem in Sect. 2 becomes the following approximate problem: Choose an admissible pair $(\sigma, \zeta)$ to minimize the cost function (3.8) subject to the switched system (3.3)-(3.5), the canonical constraints (3.6), and the continuous inequality constraints (3.7).

Let $\left(\sigma^{q, *}, \zeta^{q, *}\right)$ denote the solution of the approximate problem corresponding to $q=\left(q_{1}, \cdots, q_{p}\right)$. Furthermore, let $\left(\boldsymbol{u}^{*}, \zeta^{*}\right)$ denote the solution of the original optimal control problem. Then under suitable conditions, it can be shown that

$$
\lim _{q_{i} \rightarrow \infty} g_{0}\left(\boldsymbol{u}^{q}\left(\cdot \mid \boldsymbol{\sigma}^{q, *}\right), \boldsymbol{\zeta}^{q, *}\right)=g_{0}\left(\boldsymbol{u}^{*}, \boldsymbol{\zeta}^{*}\right)
$$

This implies that the approximate problem is a good approximation of the original problem when the number of minor subintervals is large. Proofs of (3.9) are given in $[40,45,59]$.

\subsection{Constraint Approximation}

The original optimal control problem involves an infinite number of decision variables (the value of $\boldsymbol{u}$ at every point in $[0, T]$ ) and an infinite number of constraints (the continuous inequality constraints imposed at every point in $[0, T])$. Applying the control parameterization method, in which the control is approximated by a piecewiseconstant function, causes the number of decision variables to become finite. However, control parameterization does not reduce the number of constraints. Indeed, the approximate problem still contains continuous inequality constraints that restrict the state variables at an infinite number of time points (see (3.7)). Thus, the approximate problem can be viewed as a semi-infinite programming problem [75].

In this subsection, we will discuss two popular methods for handling continuous inequality constraints in optimal control: the constraint transcription method [20, 45, $59,60]$ and the exact penalty method $[33,72,73]$. Both of these methods are designed to work in conjunction with the control parameterization technique.

In the constraint transcription method, we convert (3.7) into the following set of equivalent integral constraints:

$$
\sum_{i=1}^{p} \sum_{k=1}^{q_{i}} \int_{\gamma_{k-1}^{i}}^{\gamma_{k}^{i}} \min \left\{h_{j}\left(t, \boldsymbol{x}^{q}(t \mid \boldsymbol{\sigma}, \boldsymbol{\zeta}), \boldsymbol{\sigma}^{i, k}, \boldsymbol{\zeta}\right), 0\right\} d t=0, \quad j \in \mathcal{J}_{2}
$$


Fig. 2 The smoothing function $\varphi_{\epsilon}$ in the constraint transcription method

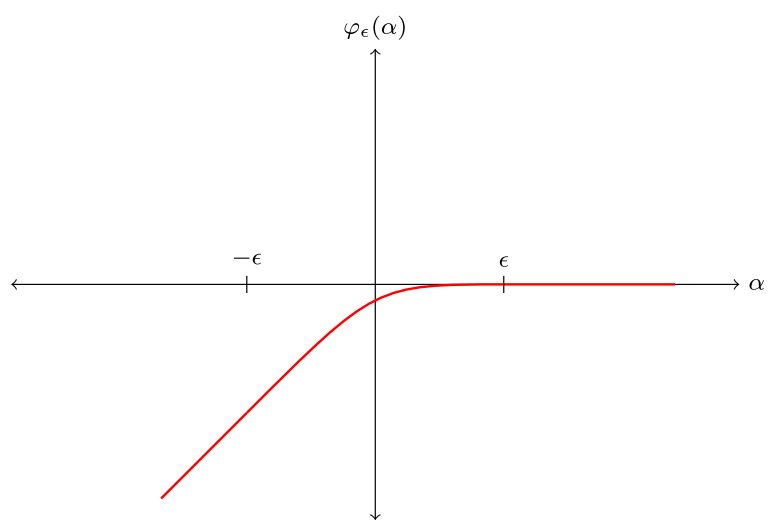

This constraint, however, is non-smooth-a non-desirable trait that poses problems for conventional optimization algorithms. Hence, we approximate (3.10) as follows:

$$
\rho+\sum_{i=1}^{p} \sum_{k=1}^{q_{i}} \int_{\gamma_{k-1}^{i}}^{\gamma_{k}^{i}} \varphi_{\epsilon}\left(h_{j}\left(t, \boldsymbol{x}^{q}(t \mid \boldsymbol{\sigma}, \zeta), \boldsymbol{\sigma}^{i, k}, \zeta\right)\right) d t \geqslant 0, \quad j \in \mathcal{J}_{2}
$$

where $\epsilon>0$ and $\rho>0$ are adjustable parameters and $\varphi_{\epsilon}: \mathbb{R} \rightarrow \mathbb{R}$ is a so-called smoothing function defined by

$$
\varphi_{\epsilon}(\alpha)= \begin{cases}\alpha, & \text { if } \alpha<-\epsilon, \\ -(\alpha-\epsilon)^{2} / 4 \epsilon, & \text { if }-\epsilon \leqslant \alpha \leqslant \epsilon, \\ 0, & \text { if } \alpha>\epsilon\end{cases}
$$

A plot of $\varphi_{\epsilon}$ is given in Fig. 2. Note that $\varphi_{\epsilon}$ is continuously differentiable and $\varphi_{\epsilon} \rightarrow$ $\min \{\cdot, 0\}$ pointwise on $\mathbb{R}$ as $\epsilon \rightarrow 0$.

It is easy to see that the approximate constraint (3.11) corresponds to a canonical inequality constraint with

$$
\Phi_{j}=\rho, \quad \mathcal{L}_{j}=\varphi_{\epsilon}\left(h_{j}\left(t, \boldsymbol{x}^{q}(t \mid \boldsymbol{\sigma}, \boldsymbol{\zeta}), \boldsymbol{\sigma}^{i, k}, \boldsymbol{\zeta}\right)\right)
$$

Thus, replacing (3.7) with (3.11) yields a dynamic optimization problem with a finite number of decision variables and a finite number of canonical constraints. It can be shown (see $[20,59]$ ) that for each $\epsilon>0$, there exists a corresponding $\rho(\epsilon)>0$ such that whenever $0<\rho<\rho(\epsilon)$, constraint (3.11) actually implies constraint (3.7). Furthermore, the optimal cost of the approximate problem (obtained by replacing (3.7) with (3.11)) converges to the true optimal cost as $\epsilon \rightarrow 0$. Hence, when $\epsilon$ and $\rho$ are sufficiently small, (3.11) is a good approximation of (3.7).

As an alternative approach, the integral term on the left-hand side of (3.11), which is always non-positive, can be appended to the system cost to form the following 
penalty function:

$$
\hat{g}_{0}^{q}(\boldsymbol{\sigma}, \boldsymbol{\zeta})=g_{0}^{q}(\boldsymbol{\sigma}, \boldsymbol{\zeta})-\omega \sum_{j \in \mathcal{J}_{2}} \sum_{i=1}^{p} \sum_{k=1}^{q_{i}} \int_{\gamma_{k-1}^{i}}^{\gamma_{k}^{i}} \varphi_{\epsilon}\left(h_{j}\left(t, \boldsymbol{x}^{q}(t \mid \boldsymbol{\sigma}, \boldsymbol{\zeta}), \boldsymbol{\sigma}^{i, k}, \boldsymbol{\zeta}\right)\right) d t
$$

where $\omega>0$ is a penalty parameter. Note that, like the approximate constraints (3.11), this penalty function is in canonical form. The corresponding penalty problem is to minimize (3.12) subject to the original canonical constraints (3.6). It can be shown (see [60]) that for each $\epsilon>0$, there exists a corresponding $\omega(\epsilon)>0$ such that for all $\omega>\omega(\epsilon)$, any solution of the penalty problem satisfies the continuous inequality constraints (3.7). Moreover, the optimal cost of the penalty problem converges to the true optimal cost as $\epsilon \rightarrow 0$, provided that $\omega$ is chosen sufficiently large (i.e. $\omega>\omega(\epsilon))$ at each step of the iteration process.

In recent years, another penalty method for handling continuous inequality constraints in optimal control—called the exact penalty method-has been developed. In the exact penalty method, we define a constraint violation function as follows:

$$
\Delta(\boldsymbol{\sigma}, \boldsymbol{\zeta}, \epsilon)=\sum_{j \in \mathcal{J}_{2}} \sum_{i=1}^{p} \sum_{k=1}^{q_{i}} \int_{\gamma_{k-1}^{i}}^{\gamma_{k}^{i}} \max \left\{-h_{j}\left(t, \boldsymbol{x}^{q}(t \mid \boldsymbol{\sigma}, \boldsymbol{\zeta}), \boldsymbol{\sigma}^{i, k}, \boldsymbol{\zeta}\right)-\epsilon \nu, 0\right\}^{2} d t
$$

where $\epsilon>0$ is a new decision variable and $v \in(0,1)$ is a fixed constant. Note that $\Delta(\sigma, \zeta, \epsilon)=0$ if and only if

$$
\begin{aligned}
& h_{j}\left(t, \boldsymbol{x}^{q}(t \mid \boldsymbol{\sigma}, \zeta), \boldsymbol{\sigma}^{i, k}, \zeta\right) \geqslant-\epsilon \nu, \\
& \quad t \in\left[\gamma_{k-1}^{i}, \gamma_{k}^{i}\right], k=1, \cdots, q_{i}, i=1, \cdots, p, j \in \mathcal{J}_{2} .
\end{aligned}
$$

Thus, $\Delta(\sigma, \zeta, \epsilon)$ measures the violation of the continuous inequality constraints (3.7).

Now, consider the following penalty function (which is in canonical form):

$$
\hat{g}_{0}^{q}(\sigma, \zeta, \epsilon)=g_{0}^{q}(\sigma, \zeta)+\epsilon^{-\alpha} \Delta(\sigma, \zeta, \epsilon)+\omega \epsilon^{\beta},
$$

where $\omega>0$ is the penalty parameter and $\alpha>0$ and $\beta>0$ are fixed constants.

In the penalty function (3.13), the last term $\omega \epsilon^{\beta}$ is designed to penalize large values of $\epsilon$, while the middle term $\epsilon^{-\alpha} \Delta(\sigma, \zeta, \epsilon)$ is designed to penalize violations in the continuous inequality constraints (3.7). When $\omega$ is large, minimizing (3.13) forces $\epsilon$ to be small, which in turn causes $\epsilon^{-\alpha}$ to become large, and thus constraint violations are penalized very severely. Hence, minimizing the penalty function for large values of $\omega$ will lead to feasible points satisfying (3.7). It can be shown that the penalty function (3.13) is "exact" in the sense that when $\omega$ is sufficiently large, any local minimizer of the penalty function is also a local minimizer of the original problem with continuous inequality constraints. See $[33,72,73]$ for details.

\subsection{Gradient Formulas}

In the previous subsection, we described three methods for approximating the continuous inequality constraints (3.7). Applying any one of these methods yields an 
approximate dynamic optimization problem with a finite number of decision variables and a finite number of canonical constraints. We now discuss how to solve this approximate problem.

In principle, the approximate problem can be viewed as a nonlinear programming problem in which a finite number of decision variables need to be chosen to minimize a given cost function subject to a set of constraints. However, this approximate problem differs from conventional nonlinear programming problems in one very important aspect: its cost and constraints are implicit, rather than explicit, functions of the decision vectors $\sigma$ and $\zeta$. Indeed, $\sigma$ and $\zeta$ influence the cost and constraints implicitly through the dynamic system (3.3)-(3.5). This means that the partial derivatives of the cost and constraints, which are required by nonlinear programming algorithms [46, 48], cannot be determined using normal differentiation rules. Thus, special methods need to be developed to determine these partial derivatives. We now describe two such methods - the variational method [31, 61, 67] and the costate method [28, 66]. These methods can be combined with any standard nonlinear programming algorithm to solve the approximate problem efficiently.

We start with the variational method. Define the Kronecker delta function as follows:

$$
\delta_{i j}= \begin{cases}1, & \text { if } i=j, \\ 0, & \text { otherwise }\end{cases}
$$

Furthermore, as is customary in the literature on control parameterization, we use the following notation for the partial derivatives of $\boldsymbol{f}^{j}$ and $\boldsymbol{\phi}^{i}$ with respect to $\boldsymbol{x}$ :

$$
\frac{\partial \boldsymbol{f}^{j}(t, \boldsymbol{x}, \boldsymbol{u}, \boldsymbol{\zeta})}{\partial \boldsymbol{x}}=\left[\begin{array}{ccc}
\frac{\partial f_{1}^{j}(t, \boldsymbol{x}, \boldsymbol{u}, \zeta)}{\partial x_{1}} & \cdots & \frac{\partial f_{1}^{j}(t, \boldsymbol{x}, \boldsymbol{u}, \zeta)}{\partial x_{n}} \\
\vdots & \ddots & \vdots \\
\frac{\partial f_{n}^{j}(t, \boldsymbol{x}, \boldsymbol{u}, \zeta)}{\partial x_{1}} & \cdots & \frac{\partial f_{n}^{j}(t, \boldsymbol{x}, \boldsymbol{u}, \zeta)}{\partial x_{n}}
\end{array}\right]
$$

and

$$
\frac{\partial \boldsymbol{\phi}^{i}(\boldsymbol{x}, \zeta)}{\partial \boldsymbol{x}}=\left[\begin{array}{ccc}
\frac{\partial \phi_{1}^{i}(\boldsymbol{x}, \zeta)}{\partial x_{1}} & \cdots & \frac{\partial \phi_{1}^{i}(\boldsymbol{x}, \zeta)}{\partial x_{n}} \\
\vdots & \ddots & \vdots \\
\frac{\partial \phi_{n}^{i}(\boldsymbol{x}, \zeta)}{\partial x_{1}} & \cdots & \frac{\partial \phi_{n}^{i}(\boldsymbol{x}, \zeta)}{\partial x_{n}}
\end{array}\right]
$$

Furthermore, the derivatives of $\Phi_{j}$ and $\mathcal{L}_{j}$ with respect to $\boldsymbol{x}$ are

$$
\begin{aligned}
\frac{\partial \Phi_{j}(\boldsymbol{x}, \boldsymbol{\zeta})}{\partial \boldsymbol{x}} & =\left[\begin{array}{lll}
\frac{\partial \Phi_{j}(\boldsymbol{x}, \boldsymbol{\zeta})}{\partial x_{1}} & \cdots & \frac{\partial \Phi_{j}(\boldsymbol{x}, \boldsymbol{\zeta})}{\partial x_{n}}
\end{array}\right] \\
\frac{\partial \mathcal{L}_{j}(t, \boldsymbol{x}, \boldsymbol{u}, \boldsymbol{\zeta})}{\partial \boldsymbol{x}} & =\left[\begin{array}{lll}
\frac{\partial \mathcal{L}_{j}(t, \boldsymbol{x}, \boldsymbol{u}, \boldsymbol{\zeta})}{\partial x_{1}} & \cdots & \frac{\partial \mathcal{L}_{j}(t, \boldsymbol{x}, \boldsymbol{u}, \boldsymbol{\zeta})}{\partial x_{n}}
\end{array}\right] .
\end{aligned}
$$

Other derivatives are defined in a similar fashion. 
Consider the following variational system corresponding to the control value $\sigma_{l}^{\varsigma, \kappa}$ :

$$
\begin{aligned}
& \dot{\boldsymbol{\xi}}^{\zeta, \kappa, l}(t)= \frac{\partial \boldsymbol{f}^{v_{i}}\left(t, \boldsymbol{x}^{q}(t \mid \boldsymbol{\sigma}, \boldsymbol{\zeta}), \boldsymbol{\sigma}^{i, k}, \boldsymbol{\zeta}\right)}{\partial \boldsymbol{x}} \boldsymbol{\xi}^{\zeta, \kappa, l}(t) \\
&+\delta_{\zeta i} \delta_{\kappa k} \frac{\partial \boldsymbol{f}^{v_{i}}\left(t, \boldsymbol{x}^{q}(t \mid \boldsymbol{\sigma}, \boldsymbol{\zeta}), \boldsymbol{\sigma}^{i, k}, \zeta\right)}{\partial u_{l}}, \\
& t \in\left[\gamma_{k-1}^{i}, \gamma_{k}^{i}\right), \quad k=\max \left\{1, \delta_{\zeta i} \kappa\right\}, \cdots, q_{i}, i=\varsigma, \cdots, p,
\end{aligned}
$$

with jump conditions

$$
\xi^{\varsigma, \kappa, l}\left(\tau_{i}^{+}\right)=\frac{\partial \boldsymbol{\phi}^{i}\left(\boldsymbol{x}^{q}\left(\tau_{i}^{-} \mid \boldsymbol{\sigma}, \zeta\right), \zeta\right)}{\partial \boldsymbol{x}} \xi^{\zeta, \kappa, l}\left(\tau_{i}^{-}\right), \quad i=\varsigma, \cdots, p-1,
$$

and initial conditions

$$
\boldsymbol{\xi}^{\zeta, \kappa, l}(t)=\mathbf{0}, \quad t \leqslant \gamma_{\kappa-1}^{\varsigma} .
$$

Let $\xi^{\zeta, \kappa, l}(\cdot \mid \sigma, \zeta)$ denote the solution of (3.14)-(3.16). Then it can be shown that $\xi^{\zeta, \kappa, l}(\cdot \mid \sigma, \zeta)$ is the partial derivative of the state with respect to the control value $\sigma_{l}^{S, \kappa}:$

$$
\frac{\partial \boldsymbol{x}^{q}(t \mid \boldsymbol{\sigma}, \zeta)}{\partial \sigma_{l}^{\zeta, \kappa}}=\boldsymbol{\xi}^{\zeta, \kappa, l}(t \mid \boldsymbol{\sigma}, \zeta), \quad t \in[0, T]
$$

See $[31,43]$ for formal proofs of this result.

Now, for the partial derivative of the state with respect to the system parameter $\zeta_{l}$, we consider another variational system:

$$
\begin{aligned}
& \dot{\boldsymbol{\psi}}^{l}(t)=\frac{\partial \boldsymbol{f}^{v_{i}}\left(t, \boldsymbol{x}^{q}(t \mid \boldsymbol{\sigma}, \zeta), \boldsymbol{\sigma}^{i, k}, \zeta\right)}{\partial \boldsymbol{x}} \boldsymbol{\psi}^{l}(t)+\frac{\partial \boldsymbol{f}^{v_{i}}\left(t, \boldsymbol{x}^{q}(t \mid \boldsymbol{\sigma}, \zeta), \boldsymbol{\sigma}^{i, k}, \zeta\right)}{\partial \zeta_{l}} \\
& \quad t \in\left[\gamma_{k-1}^{i}, \gamma_{k}^{i}\right), k=1, \cdots, q_{i}, i=1, \cdots, p
\end{aligned}
$$

with jump conditions

$$
\boldsymbol{\psi}^{l}\left(\tau_{i}^{+}\right)=\frac{\partial \boldsymbol{\phi}^{i}\left(\boldsymbol{x}^{q}\left(\tau_{i}^{-} \mid \boldsymbol{\sigma}, \zeta\right), \zeta\right)}{\partial \boldsymbol{x}} \boldsymbol{\psi}^{l}\left(\tau_{i}^{-}\right)+\frac{\partial \boldsymbol{\phi}^{i}\left(\boldsymbol{x}^{q}\left(\tau_{i}^{-} \mid \boldsymbol{\sigma}, \zeta\right), \zeta\right)}{\partial \zeta_{l}}, \quad i=1, \cdots, p-1
$$

and initial conditions

$$
\boldsymbol{\psi}^{l}(0)=\mathbf{0} .
$$

Let $\boldsymbol{\psi}^{l}(\cdot \mid \boldsymbol{\sigma}, \zeta)$ denote the solution of (3.18)-(3.20). Then according to the results in $[31,43]$,

$$
\frac{\partial \boldsymbol{x}^{q}(t \mid \boldsymbol{\sigma}, \zeta)}{\partial \zeta_{l}}=\boldsymbol{\psi}^{l}(t \mid \boldsymbol{\sigma}, \zeta), \quad t \in[0, T]
$$

Equations (3.17) and (3.21) can be used to determine the partial derivatives of the cost and constraints in the approximate problem. Recall that the cost and constraints 
have the following canonical form:

$$
g_{j}^{q}(\boldsymbol{\sigma}, \boldsymbol{\zeta})=\Phi_{j}\left(\boldsymbol{x}^{q}(T \mid \boldsymbol{\sigma}, \boldsymbol{\zeta}), \boldsymbol{\zeta}\right)+\sum_{i=1}^{p} \sum_{k=1}^{q_{i}} \int_{\gamma_{k-1}^{i}}^{\gamma_{k}^{i}} \mathcal{L}_{j}\left(t, \boldsymbol{x}^{q}(t \mid \boldsymbol{\sigma}, \boldsymbol{\zeta}), \boldsymbol{\sigma}^{i, k}, \boldsymbol{\zeta}\right) d t
$$

By differentiating this canonical form with respect to $\sigma_{l}^{\zeta, \kappa}$, and then applying (3.17), we obtain the following gradient formulas:

$$
\begin{aligned}
\frac{\partial g_{j}^{q}(\boldsymbol{\sigma}, \boldsymbol{\zeta})}{\partial \sigma_{l}^{\zeta, \kappa}}= & \frac{\partial \Phi_{j}\left(\boldsymbol{x}^{q}(T), \boldsymbol{\zeta}\right)}{\partial \boldsymbol{x}} \boldsymbol{\xi}^{\zeta, \kappa, l}(T) \\
& +\sum_{i=1}^{p} \sum_{k=1}^{q_{i}} \int_{\gamma_{k-1}^{i}}^{\gamma_{k}^{i}}\left\{\frac{\partial \mathcal{L}_{j}\left(t, \boldsymbol{x}^{q}(t), \boldsymbol{\sigma}^{i, k}, \boldsymbol{\zeta}\right)}{\partial \boldsymbol{x}} \boldsymbol{\xi}^{\zeta, \kappa, l}(t)\right. \\
& \left.+\delta_{\zeta i} \delta_{\kappa k} \frac{\partial \mathcal{L}_{j}\left(t, \boldsymbol{x}^{q}(t), \boldsymbol{\sigma}^{i, k}, \zeta\right)}{\partial u_{l}}\right\} d t,
\end{aligned}
$$

where $\boldsymbol{x}^{q}(\cdot)=\boldsymbol{x}^{q}(\cdot \mid \boldsymbol{\sigma}, \boldsymbol{\zeta})$ and $\boldsymbol{\xi}^{\zeta, \kappa, l}(\cdot)=\boldsymbol{\xi}^{\zeta, \kappa, l}(\cdot \mid \boldsymbol{\sigma}, \boldsymbol{\zeta})$. Similarly,

$$
\begin{aligned}
\frac{\partial g_{j}^{q}(\boldsymbol{\sigma}, \boldsymbol{\zeta})}{\partial \zeta_{l}}= & \frac{\partial \Phi_{j}\left(\boldsymbol{x}^{q}(T), \boldsymbol{\zeta}\right)}{\partial \boldsymbol{x}} \boldsymbol{\psi}^{l}(T)+\frac{\partial \Phi_{j}\left(\boldsymbol{x}^{q}(T), \boldsymbol{\zeta}\right)}{\partial \zeta_{l}} \\
& +\sum_{i=1}^{p} \sum_{k=1}^{q_{i}} \int_{\gamma_{k-1}^{i}}^{\gamma_{k}^{i}}\left\{\frac{\partial \mathcal{L}_{j}\left(t, \boldsymbol{x}^{q}(t), \boldsymbol{\sigma}^{i, k}, \boldsymbol{\zeta}\right)}{\partial \boldsymbol{x}} \boldsymbol{\psi}^{l}(t)\right. \\
& \left.+\frac{\partial \mathcal{L}_{j}\left(t, \boldsymbol{x}^{q}(t), \boldsymbol{\sigma}^{i, k}, \boldsymbol{\zeta}\right)}{\partial \zeta_{l}}\right\} d t
\end{aligned}
$$

where $\boldsymbol{x}^{q}(\cdot)=\boldsymbol{x}^{q}(\cdot \mid \boldsymbol{\sigma}, \boldsymbol{\zeta})$ and $\boldsymbol{\psi}^{l}(\cdot)=\boldsymbol{\psi}^{l}(\cdot \mid \boldsymbol{\sigma}, \zeta)$. Thus, the gradients of the cost and constraints in the approximate problem can be computed by solving the variational systems (3.14)-(3.16) and (3.18)-(3.20) in conjunction with the state system (3.3)(3.5). This procedure can be combined with a standard nonlinear optimization algorithm, such as sequential quadratic programming $[46,48]$, to solve the approximate problem efficiently.

A disadvantage with the variational method is that it requires solving a large number of differential equations - there are $q_{1} r+q_{2} r+\cdots+q_{p} r$ variational systems in the form of (3.14)-(3.16) and $w$ variational systems in the form of (3.18)-(3.20), with each variational system of dimension $n$. The costate method is an alternative to the variational method that (in most cases) involves solving fewer differential equations. In the costate method, we define the following costate system corresponding to the $j$ th canonical form:

$$
\begin{gathered}
\dot{\lambda}^{j}(t)=-\left[\frac{\partial \mathcal{L}_{j}\left(t, \boldsymbol{x}^{q}(t \mid \boldsymbol{\sigma}, \boldsymbol{\zeta}), \boldsymbol{\sigma}^{i, k}, \boldsymbol{\zeta}\right)}{\partial \boldsymbol{x}}\right]^{\top}-\left[\frac{\partial \boldsymbol{f}^{v_{i}}\left(t, \boldsymbol{x}^{q}(t \mid \boldsymbol{\sigma}, \boldsymbol{\zeta}), \boldsymbol{\sigma}^{i, k}, \boldsymbol{\zeta}\right)}{\partial \boldsymbol{x}}\right]^{\top} \lambda^{j}(t), \\
t \in\left[\gamma_{k-1}^{i}, \gamma_{k}^{i}\right), k=1, \cdots, q_{i}, i=1, \cdots, p,
\end{gathered}
$$


with terminal condition

$$
\lambda^{j}(T)=\left[\frac{\partial \Phi_{j}\left(\boldsymbol{x}^{q}(T \mid \boldsymbol{\sigma}, \zeta), \zeta\right)}{\partial \boldsymbol{x}}\right]^{\top}
$$

As with the variational systems, each costate system is of dimension $n$. Let $\lambda^{j}(\cdot \mid \sigma, \zeta)$ denote the solution of the costate system (3.24)-(3.25). Then

$$
\frac{\partial g_{j}^{q}(\boldsymbol{\sigma}, \boldsymbol{\zeta})}{\partial \sigma_{l}^{\varsigma, \kappa}}=\int_{\gamma_{\kappa-1}^{\varsigma}}^{\gamma_{\kappa}^{\varsigma}}\left\{\frac{\partial \mathcal{L}_{j}\left(t, \boldsymbol{x}^{q}(t), \boldsymbol{\sigma}^{\varsigma, \kappa}, \boldsymbol{\zeta}\right)}{\partial u_{l}}+\lambda^{j}(t)^{\top} \frac{\partial \boldsymbol{f}^{v_{\varsigma}}\left(t, \boldsymbol{x}^{q}(t), \boldsymbol{\sigma}^{\varsigma, \kappa}, \boldsymbol{\zeta}\right)}{\partial u_{l}}\right\} d t
$$

and

$$
\begin{aligned}
\frac{\partial g_{j}^{q}(\boldsymbol{\sigma}, \boldsymbol{\zeta})}{\partial \zeta_{l}}= & \frac{\partial \Phi_{j}\left(\boldsymbol{x}^{q}(T), \boldsymbol{\zeta}\right)}{\partial \zeta_{l}}+\sum_{i=1}^{p} \sum_{k=1}^{q_{i}} \int_{\gamma_{k-1}^{i}}^{\gamma_{k}^{i}}\left\{\frac{\partial \mathcal{L}_{j}\left(t, \boldsymbol{x}^{q}(t), \boldsymbol{\sigma}^{i, k}, \boldsymbol{\zeta}\right)}{\partial \zeta_{l}}\right. \\
& \left.+\lambda^{j}(t)^{\top} \frac{\partial \boldsymbol{f}^{v_{i}}\left(t, \boldsymbol{x}^{q}(t), \boldsymbol{\sigma}^{i, k}, \boldsymbol{\zeta}\right)}{\partial \zeta_{l}}\right\} d t
\end{aligned}
$$

where $\boldsymbol{x}^{q}(\cdot)=\boldsymbol{x}^{q}(\cdot \mid \boldsymbol{\sigma}, \boldsymbol{\zeta})$ and $\lambda^{j}(\cdot)=\lambda^{j}(\cdot \mid \boldsymbol{\sigma}, \zeta)$. These gradient formulas are proved in [59]. By incorporating these formulas into a gradient-based optimization method, the approximate problem can be solved as a nonlinear programming problem.

\section{Variable Switching Times}

In Sect. 3, we assumed that both the control switching times and the subsystem switching times are fixed. The more common scenario in practice is that these switching times are actually decision variables to be optimized. In this case, the state trajectory should be denoted as follows:

$$
\boldsymbol{x}^{q}(\cdot)=\boldsymbol{x}^{q}(\cdot \mid \boldsymbol{\sigma}, \boldsymbol{\gamma}, \boldsymbol{\zeta})
$$

where

$$
\boldsymbol{\gamma}=\left[\left(\boldsymbol{\gamma}^{1}\right)^{\top}, \cdots,\left(\boldsymbol{\gamma}^{p}\right)^{\top}\right]^{\top}
$$

and

$$
\boldsymbol{\gamma}^{i}=\left[\gamma_{1}^{i}, \cdots, \gamma_{q_{i}}^{i}\right]^{\top}, \quad i=1, \cdots, p .
$$

This modified notation emphasizes that the switching times, along with the control and parameter vectors, influence the evolution of the state trajectory.

Since the switching times are now decision variables, the following constraints must be imposed in the approximate problem:

$$
\gamma_{0}^{i} \leqslant \gamma_{1}^{i} \leqslant \cdots \leqslant \gamma_{q_{i}-1}^{i} \leqslant \gamma_{q_{i}}^{i}, \quad i=1, \cdots, p,
$$

and

$$
\gamma_{q_{p}}^{p}=T
$$


where, as in Sect. 3, $\gamma_{0}^{i}=\tau_{i-1}$ and $\gamma_{q_{i}}^{i}=\tau_{i}$.

To solve the approximate problem when the switching times are variable, the gradients of the cost and constraints with respect to the switching times are required. Variational and costate formulas for computing these gradients are derived in [29]. In principle, these gradient formulas can be combined with the gradient formulas for the control and parameter vectors in Sect. 3 to solve the approximate problem via nonlinear optimization techniques. This approach, however, suffers from the following drawbacks:

(1) The partial derivatives of the canonical functions with respect to the switching times only exist when the switching times are distinct (hence, problems will arise if the optimal solution involves "deleting" certain non-optimal subintervals); and

(2) It is cumbersome to integrate the state and variational/costate systems numerically when the switching times are variable.

These issues are examined in detail in [29]. To avoid the problems caused by variable switching times, the so-called time-scaling transformation [15, 28, 39, 66] can be applied. This transformation, called the control parameterization enhancing transform (CPET) in earlier work, is now one of the most popular tools for optimizing switching times in dynamic optimization problems.

\subsection{The Time-Scaling Transformation: Version 1}

For simplicity, we assume that there are no state jumps. Thus,

$$
\boldsymbol{\phi}^{i}\left(\boldsymbol{x}\left(\tau_{i}^{-}\right), \zeta\right)=\boldsymbol{x}\left(\tau_{i}^{-}\right), \quad i=1, \cdots, p-1 .
$$

Let $\theta_{k}^{i}$ denote the duration of the $k$ th minor subinterval in $\left[\tau_{i-1}, \tau_{i}\right)$. That is,

$$
\theta_{k}^{i}=\gamma_{k}^{i}-\gamma_{k-1}^{i}, \quad k=1, \cdots, q_{i}, i=1, \cdots, p .
$$

Furthermore, define

$$
\tilde{s}_{k}^{i}=\sum_{z=1}^{i-1} q_{z}+k, \quad k=0, \cdots, q_{i}, i=1, \cdots, p,
$$

where $q_{i}, i=1, \cdots, p$, are as defined in Sect. 3. Note that

$$
\left\{\tilde{s}_{k}^{i}: k=0, \cdots, q_{i}, i=1, \cdots, p\right\}=\left\{0,1,2, \cdots, q_{1}+q_{2}+\cdots+q_{p}\right\} .
$$

The time-scaling transformation works by introducing a new time variable $s \in$ $\left[0, q_{1}+\cdots+q_{p}\right]$ and relating $s$ to $t$ through the equation $t=\mu(s)$, where $\mu$ is the so-called time-scaling function defined by

$$
\begin{gathered}
\mu(s)=\sum_{z=1}^{i-1} \sum_{l=1}^{q_{z}} \theta_{l}^{z}+\sum_{l=1}^{k-1} \theta_{l}^{i}+\theta_{k}^{i}\left(s-\tilde{s}_{k-1}^{i}\right), \\
s \in\left[\tilde{s}_{k-1}^{i}, \tilde{s}_{k}^{i}\right], k=1, \cdots, q_{i}, i=1, \cdots, p .
\end{gathered}
$$




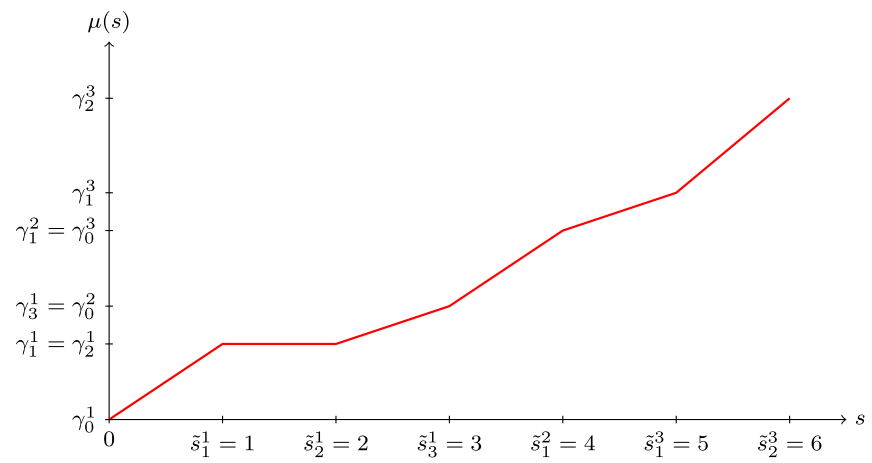

Fig. 3 An example of the time-scaling function for $p=3$ and $q=(3,1,2)$. Note that $\tilde{s}_{3}^{1}=\tilde{s}_{0}^{2}=3$ and $\tilde{s}_{1}^{2}=\tilde{s}_{0}^{3}=4$

An example of the time-scaling function is given in Fig. 3. Note that the time-scaling function is piecewise-linear, continuous, and non-decreasing. These are three fundamental properties of the time-scaling function. Furthermore,

$$
\frac{d \mu(s)}{d s}=\theta_{k}^{i}, \quad s \in\left(\tilde{s}_{k-1}^{i}, \tilde{s}_{k}^{i}\right), k=1, \cdots, q_{i}, i=1, \cdots, p .
$$

The new decision parameters $\theta_{k}^{i}, k=1, \cdots, q_{i}, i=1, \cdots, p$, satisfy the following constraints:

$$
0 \leqslant \theta_{k}^{i} \leqslant T, \quad k=1, \cdots, q_{i}, i=1, \cdots, p,
$$

and

$$
\sum_{i=1}^{p} \sum_{k=1}^{q_{i}} \theta_{k}^{i}=T
$$

Let

$$
\boldsymbol{\theta}^{i}=\left[\theta_{1}^{i}, \cdots, \theta_{q_{i}}^{i}\right]^{\top}, \quad i=1, \cdots, p,
$$

and

$$
\boldsymbol{\theta}=\left[\left(\boldsymbol{\theta}^{1}\right)^{\top}, \cdots,\left(\boldsymbol{\theta}^{p}\right)^{\top}\right]^{\top} .
$$

Any $\boldsymbol{\theta} \in \mathbb{R}^{q_{1}+\cdots+q_{p}}$ satisfying (4.1) and (4.2) is called an admissible duration vector.

Now, since $\tilde{s}_{k}^{i}-\tilde{s}_{k-1}^{i}=1$ for $k \geqslant 1$,

$$
\mu\left(\tilde{s}_{k}^{i}\right)=\sum_{z=1}^{i-1} \sum_{l=1}^{q_{z}} \theta_{l}^{z}+\sum_{l=1}^{k} \theta_{l}^{i}=\gamma_{k}^{i}, \quad k=1, \cdots, q_{i}, i=1, \cdots, p .
$$

Thus, the time-scaling function maps the fixed integer $s=\tilde{s}_{k}^{i}$ to the switching time $t=\gamma_{k}^{i}$ 
Let $\tilde{\boldsymbol{x}}^{q}(s)=\boldsymbol{x}^{q}(\mu(s))$. If $s \in\left[\tilde{s}_{k-1}^{i}, \tilde{s}_{k}^{i}\right]$, then $\mu(s) \in\left[\gamma_{k-1}^{i}, \gamma_{k}^{i}\right]$, and thus

$$
\begin{aligned}
\dot{\tilde{\boldsymbol{x}}}^{q}(s) & =\frac{d}{d s}\left\{\boldsymbol{x}^{q}(\mu(s))\right\} \\
& =\theta_{k}^{i} \boldsymbol{f}^{v_{i}}\left(\mu(s), \boldsymbol{x}^{q}(\mu(s)), \boldsymbol{\sigma}^{i, k}, \zeta\right)=\theta_{k}^{i} \boldsymbol{f}^{v_{i}}\left(\mu(s), \tilde{\boldsymbol{x}}^{q}(s), \boldsymbol{\sigma}^{i, k}, \boldsymbol{\zeta}\right) .
\end{aligned}
$$

In these new dynamics, mode switches occur at the fixed integers $s=\tilde{s}_{k}^{i}, k=$ $1, \cdots, q_{i}, i=1, \cdots, p$.

Since $\mu(0)=0$, the initial condition (3.5) becomes

$$
\tilde{\boldsymbol{x}}^{q}(0)=\boldsymbol{x}^{q}(\mu(0))=\boldsymbol{x}^{q}(0)=\boldsymbol{x}^{0} .
$$

Combining (4.3) and (4.4), we obtain the following new switched system:

$$
\begin{aligned}
& \dot{\tilde{\boldsymbol{x}}}^{q}(s)=\theta_{k}^{i} \boldsymbol{f}^{v_{i}}\left(\mu(s), \tilde{\boldsymbol{x}}^{q}(s), \boldsymbol{\sigma}^{i, k}, \zeta\right), \quad s \in\left[\tilde{s}_{k-1}^{i}, \tilde{s}_{k}^{i}\right), k=1, \cdots, q_{i}, i=1, \cdots, p, \\
& \tilde{\boldsymbol{x}}^{q}(0)=\boldsymbol{x}^{0} .
\end{aligned}
$$

Let $\tilde{\boldsymbol{x}}^{q}(\cdot \mid \boldsymbol{\sigma}, \boldsymbol{\theta}, \boldsymbol{\zeta})$ denote the solution of this new switched system corresponding to the admissible triple $(\boldsymbol{\sigma}, \boldsymbol{\theta}, \boldsymbol{\zeta})$. Then constraints (3.6) and (3.7) become

$$
\begin{aligned}
& \Phi_{j}\left(\tilde{\boldsymbol{x}}^{q}\left(\tilde{s}_{q_{p}}^{p} \mid \boldsymbol{\sigma}, \boldsymbol{\theta}, \boldsymbol{\zeta}\right), \boldsymbol{\zeta}\right) \\
& \quad+\sum_{i=1}^{p} \sum_{k=1}^{q_{i}} \int_{\tilde{s}_{k-1}^{i}}^{\tilde{s}_{k}^{i}} \theta_{k}^{i} \mathcal{L}_{j}\left(\mu(s), \tilde{\boldsymbol{x}}^{q}(s \mid \boldsymbol{\sigma}, \boldsymbol{\theta}, \boldsymbol{\zeta}), \boldsymbol{\sigma}^{i, k}, \zeta\right) d s\left\{\begin{array}{l}
=0 \\
\geqslant 0
\end{array} \quad j \in \mathcal{J}_{1},\right.
\end{aligned}
$$

and

$$
\begin{aligned}
& \quad h_{j}\left(\mu(s), \tilde{\boldsymbol{x}}^{q}(s \mid \boldsymbol{\sigma}, \boldsymbol{\theta}, \boldsymbol{\zeta}), \boldsymbol{\sigma}^{i, k}, \boldsymbol{\zeta}\right) \geqslant 0, \\
& \quad \quad s \in\left[\tilde{s}_{k-1}^{i}, \tilde{s}_{k}^{i}\right], k=1, \cdots, q_{i}, i=1, \cdots, p, j \in \mathcal{J}_{2} .
\end{aligned}
$$

Similarly, the cost function (3.8) becomes

$$
\begin{aligned}
\tilde{g}_{0}^{q}(\boldsymbol{\sigma}, \boldsymbol{\theta}, \boldsymbol{\zeta})= & \Phi_{0}\left(\tilde{\boldsymbol{x}}^{q}\left(\tilde{s}_{q_{p}}^{p} \mid \boldsymbol{\sigma}, \boldsymbol{\theta}, \boldsymbol{\zeta}\right), \boldsymbol{\zeta}\right) \\
& +\sum_{i=1}^{p} \sum_{k=1}^{q_{i}} \int_{\tilde{s}_{k-1}^{i}}^{\tilde{s}_{k}^{i}} \theta_{k}^{i} \mathcal{L}_{0}\left(\mu(s), \tilde{\boldsymbol{x}}^{q}(s \mid \boldsymbol{\sigma}, \boldsymbol{\theta}, \boldsymbol{\zeta}), \boldsymbol{\sigma}^{i, k}, \zeta\right) d s
\end{aligned}
$$

Thus, after applying the time-scaling transformation, we obtain the following equivalent problem: Choose an admissible triple $(\sigma, \boldsymbol{\theta}, \boldsymbol{\zeta})$ to minimize the cost function (4.9) subject to the switched system (4.5)-(4.6), the canonical constraints (4.7), and the continuous inequality constraints (4.8).

Note that the time-scaling transformation has replaced the variable switching times in the original approximate problem with conventional decision parameters in the equivalent problem. Since the switching times in the equivalent problem are fixed, this problem can be solved readily by combining the gradient formulas in Sect. 3 with any standard gradient-based optimization method. See [28, 31, 53] for more details. 


\subsection{The Time-Scaling Transformation: Version 2}

There is another way of applying the time-scaling transformation. This alternative approach, which is described in references [34, 39, 66], involves transforming the switched system with variable switching times into an enlarged system of ordinary differential equations subject to boundary constraints.

First, define the following new state variables:

$$
\boldsymbol{y}^{i, k}(s)=\boldsymbol{x}^{q}\left(\gamma_{k-1}^{i}+\theta_{k}^{i} s\right), \quad s \in[0,1], k=1, \cdots, q_{i}, i=1, \cdots, p,
$$

where, as in the previous subsection, $\theta_{k}^{i}=\gamma_{k}^{i}-\gamma_{k-1}^{i}$ and

$$
\gamma_{k-1}^{i}=\sum_{z=1}^{i-1} \sum_{l=1}^{q_{z}} \theta_{l}^{z}+\sum_{l=1}^{k-1} \theta_{l}^{i} .
$$

Note that $\boldsymbol{y}^{i, k}(\cdot)$ corresponds to $\boldsymbol{x}^{q}(\cdot)$ on the subinterval $\left[\gamma_{k-1}^{i}, \gamma_{k}^{i}\right]$.

Differentiating (4.10) with respect to $s$ yields

$$
\begin{aligned}
\dot{\boldsymbol{y}}^{i, k}(s) & =\frac{d}{d s}\left\{\boldsymbol{y}^{i, k}(s)\right\} \\
& =\frac{d}{d s}\left\{\boldsymbol{x}^{q}\left(\gamma_{k-1}^{i}+\theta_{k}^{i} s\right)\right\}=\theta_{k}^{i} \boldsymbol{f}^{v_{i}}\left(\gamma_{k-1}^{i}+\theta_{k}^{i} s, \boldsymbol{y}^{i, k}(s), \boldsymbol{\sigma}^{i, k}, \zeta\right), \quad s \in[0,1] .
\end{aligned}
$$

Furthermore,

$$
\boldsymbol{y}^{i, k}(1)=\boldsymbol{x}^{q}\left(\gamma_{k}^{i}\right)=\boldsymbol{y}^{i, k+1}(0), \quad k=1, \cdots, q_{i}-1, i=1, \cdots, p,
$$

and

$$
\boldsymbol{y}^{i, q_{i}}(1)=\boldsymbol{x}^{q}\left(\gamma_{q_{i}}^{i}\right)=\boldsymbol{x}^{q}\left(\gamma_{0}^{i+1}\right)=\boldsymbol{y}^{i+1,1}(0), \quad i=1, \cdots, p-1 .
$$

Equations (4.12) and (4.13) are boundary conditions expressing the initial value of one state variable in terms of the final value of another. The initial conditions (3.5) become

$$
\boldsymbol{y}^{1,1}(0)=\boldsymbol{x}^{q}(0)=\boldsymbol{x}^{0} .
$$

Let $\boldsymbol{y}^{i, k}(\cdot \mid \boldsymbol{\sigma}, \boldsymbol{\theta}, \zeta), k=1, \cdots, q_{i}, i=1, \cdots, p$, denote the solution of (4.11)-(4.14). Then the canonical constraints (3.6) become

$$
\begin{aligned}
& \Phi_{j}\left(\boldsymbol{y}^{p, q_{p}}(1 \mid \boldsymbol{\sigma}, \boldsymbol{\theta}, \boldsymbol{\zeta}), \boldsymbol{\zeta}\right) \\
& \quad+\sum_{i=1}^{p} \sum_{k=1}^{q_{i}} \int_{0}^{1} \theta_{k}^{i} \tilde{\mathcal{L}}_{j}\left(s, \boldsymbol{y}^{i, k}(s \mid \boldsymbol{\sigma}, \boldsymbol{\theta}, \boldsymbol{\zeta}), \boldsymbol{\sigma}^{i, k}, \zeta\right) d s\left\{\begin{array}{l}
=0 \\
\geqslant 0
\end{array} \quad j \in \mathcal{J}_{1},\right.
\end{aligned}
$$

where

$$
\tilde{\mathcal{L}}_{j}\left(s, \boldsymbol{y}^{i, k}(s \mid \boldsymbol{\sigma}, \boldsymbol{\theta}, \boldsymbol{\zeta}), \boldsymbol{\sigma}^{i, k}, \boldsymbol{\zeta}\right)=\mathcal{L}_{j}\left(\gamma_{k-1}^{i}+\theta_{k}^{i} s, \boldsymbol{y}^{i, k}(s \mid \boldsymbol{\sigma}, \boldsymbol{\theta}, \boldsymbol{\zeta}), \boldsymbol{\sigma}^{i, k}, \boldsymbol{\zeta}\right) .
$$


Similarly, the continuous inequality constraints (3.7) become

$\tilde{h}_{j}\left(s, \boldsymbol{y}^{i, k}(s \mid \boldsymbol{\sigma}, \boldsymbol{\theta}, \zeta), \boldsymbol{\sigma}^{i, k}, \zeta\right) \geqslant 0, \quad s \in[0,1], k=1, \cdots, q_{i}, i=1, \cdots, p, j \in \mathcal{J}_{2}$,

where

$$
\tilde{h}_{j}\left(s, \boldsymbol{y}^{i, k}(s \mid \boldsymbol{\sigma}, \boldsymbol{\theta}, \zeta), \boldsymbol{\sigma}^{i, k}, \zeta\right)=h_{j}\left(\gamma_{k-1}^{i}+\theta_{k}^{i} s, \boldsymbol{y}^{i, k}(s \mid \boldsymbol{\sigma}, \boldsymbol{\theta}, \zeta), \boldsymbol{\sigma}^{i, k}, \zeta\right)
$$

Moreover, the cost function (3.8) becomes

$$
\begin{aligned}
\tilde{g}_{0}^{q}(\boldsymbol{\sigma}, \boldsymbol{\theta}, \boldsymbol{\zeta})= & \Phi_{0}\left(\boldsymbol{y}^{p, q_{p}}(1 \mid \boldsymbol{\sigma}, \boldsymbol{\theta}, \boldsymbol{\zeta}), \boldsymbol{\zeta}\right) \\
& +\sum_{i=1}^{p} \sum_{k=1}^{q_{i}} \int_{0}^{1} \theta_{k}^{i} \tilde{\mathcal{L}}_{0}\left(s, \boldsymbol{y}^{i, k}(s \mid \boldsymbol{\sigma}, \boldsymbol{\theta}, \boldsymbol{\zeta}), \boldsymbol{\sigma}^{i, k}, \zeta\right) d s
\end{aligned}
$$

The equivalent problem is: Choose an admissible triple $(\boldsymbol{\sigma}, \boldsymbol{\theta}, \boldsymbol{\zeta})$ to minimize the cost function (4.17) subject to the boundary value problem (4.11)-(4.14), the canonical constraints (4.15), and the continuous inequality constraints (4.16). This is a standard dynamic optimization problem with fixed time horizon $[0,1]$. Such problems can be solved readily using existing methods.

\subsection{State Jumps and the Time-Scaling Transformation}

Thus far in this section, we have ignored the possibility of state jumps occurring at the switching times. This is because state jumps cause complications when applying the time-scaling transformation. To see why, consider a situation in which two adjacent switching times coincide (this occurs when the optimization procedure decides to remove a "non-optimal" subsystem by merging its start and end times). In this case, there should be a single state jump occurring at the common switching time. However, under the time-scaling transformation, two switching times that coincide in the original time horizon are still treated as separate switching times in the new time horizon. Thus, two state jumps will be imposed instead of one. For example, if $\tau_{1}=\tau_{2}$ (i.e. stage 2 has been removed), then the time-scaling transformation (version 1) will map $t=\tau_{1}$ to $s=q_{1}$, and $t=\tau_{2}$ to $s=q_{1}+q_{2}$. Thus, in the new time horizon, there will be a state jump at $s=q_{1}$ and another state jump at $s=q_{1}+q_{2}$ (note that $\theta_{1}^{2}=\theta_{2}^{2}=\cdots=\theta_{q_{2}}^{2}=0$ here, so the state vector will be constant on the open interval $\left.\left(q_{1}, q_{1}+q_{2}\right)\right)$. This corresponds to a "double jump" in the original time horizon at the common switching time $t=\tau_{1}=\tau_{2}$.

To explain further, recall the state jump conditions from Sect. 2:

$$
x\left(\tau_{i}^{+}\right)=\phi^{i}\left(x\left(\tau_{i}^{-}\right), \zeta\right), \quad i=1, \cdots, p-1 .
$$

After applying control parameterization, the switching time $\tau_{i}$ is replaced by $\gamma_{q_{i}}^{i}$, where $q_{i}$ is the number of minor subintervals in $\left[\tau_{i-1}, \tau_{i}\right)$. Thus, the state jump conditions become

$$
\lim _{t \rightarrow \gamma_{q_{i}}^{i}+} \boldsymbol{x}^{q}(t)=\lim _{t \rightarrow \gamma_{q_{i}}^{i}-} \boldsymbol{\phi}^{i}\left(\boldsymbol{x}^{q}(t), \boldsymbol{\zeta}\right), \quad i=1, \cdots, p-1,
$$


where $\boldsymbol{x}^{q}(\cdot)=\boldsymbol{x}^{q}(\cdot \mid \boldsymbol{\sigma}, \boldsymbol{\gamma}, \boldsymbol{\zeta})$. Recall that the time-scaling transformation maps $t=\gamma_{q_{i}}^{i}$ to the fixed integer $s=\tilde{s}_{q_{i}}^{i}=q_{1}+\cdots+q_{i}$. Recall also that the time-scaling function $\mu$ is continuous and non-decreasing, so $\mu(s) \rightarrow \tau_{i} \pm$ as $s \rightarrow\left(q_{1}+\cdots+q_{i}\right) \pm$. Thus, by applying version 1 of time-scaling transformation to the jump conditions (4.19), we obtain

$$
\lim _{s \rightarrow\left(q_{1}+\cdots+q_{i}\right)+} \tilde{\boldsymbol{x}}^{q}(s)=\lim _{s \rightarrow\left(q_{1}+\cdots+q_{i}\right)-} \boldsymbol{\phi}^{i}\left(\tilde{\boldsymbol{x}}^{q}(s), \zeta\right), \quad i=1, \cdots, p-1,
$$

where $\tilde{\boldsymbol{x}}^{q}(s)=\boldsymbol{x}^{q}(\mu(s))$. Alternatively, using version 2 of the time-scaling transformation, since $\boldsymbol{y}^{i+1,1}(0)$ corresponds to $\boldsymbol{x}^{q}\left(\tau_{i}^{+}\right)$and $\boldsymbol{y}^{i, q_{i}}(1)$ corresponds to $\boldsymbol{x}^{q}\left(\tau_{i}^{-}\right)$, the jump conditions (4.19) become

$$
\boldsymbol{y}^{i+1,1}(0)=\boldsymbol{x}^{q}\left(\tau_{i}^{+}\right)=\boldsymbol{\phi}^{i}\left(\boldsymbol{x}^{q}\left(\tau_{i}^{-}\right), \zeta\right)=\boldsymbol{\phi}^{i}\left(\boldsymbol{y}^{i, q_{i}}(1), \zeta\right), \quad i=1, \cdots, p-1 .
$$

It is important to realize that (4.20) and (4.21) are imposed for every $i=1, \cdots, p-1$. Thus, in the new time horizon, there are exactly $p-1$ state jumps. However, in the original time horizon, there may be less than $p-1$ state jumps; it is possible that several switching times coincide, with only a single state jump occurring at the common switching time. It follows that under the time-scaling transformation, a single state jump could be mapped into several state jumps. This may be acceptable in situations where it makes sense to allow such "multi-jumps", but in other situations it may not be an accurate reflection of the real system under consideration.

In fact, the definition of the state jump conditions (4.18) is ambiguous when two or more switching times coincide. To see why, suppose that $\tau_{i}=\tau_{i-1}$. Then

$$
\boldsymbol{x}\left(\tau_{i}^{+}\right)=\boldsymbol{\phi}^{i}\left(\boldsymbol{x}\left(\tau_{i}^{-}\right), \zeta\right)
$$

and

$$
\boldsymbol{x}\left(\tau_{i}^{+}\right)=\boldsymbol{x}\left(\tau_{i-1}^{+}\right)=\boldsymbol{\phi}^{i-1}\left(\boldsymbol{x}\left(\tau_{i-1}^{-}\right), \zeta\right)=\boldsymbol{\phi}^{i-1}\left(\boldsymbol{x}\left(\tau_{i}^{-}\right), \zeta\right) .
$$

Thus, we have two different equations describing the state jump at the common switching time $\tau_{i}=\tau_{i-1}$ : one equation involves $\boldsymbol{\phi}^{i}$; the other involves $\boldsymbol{\phi}^{i-1}$. This ambiguity can be avoided by following the approach proposed in [8, 42], which involves replacing (4.18) with

$$
\boldsymbol{x}\left(\tau_{i}^{+}\right)=\boldsymbol{\phi}^{i}\left(\boldsymbol{x}\left(\tau_{i}^{-}\right), \zeta\right), \quad i \in \mathcal{I}
$$

where

$$
\mathcal{I}=\left\{i \in\{1, \cdots, p-1\}: \tau_{i}>\tau_{i-1}\right\} .
$$

This ensures that state jumps are imposed at each distinct switching time; "multijumps" are therefore not permitted. Applying version 1 of the time-scaling transformation to (4.22) yields

$$
\lim _{s \rightarrow\left(q_{1}+\cdots+q_{i}\right)+} \tilde{\boldsymbol{x}}^{q}(s)= \begin{cases}\lim _{s \rightarrow\left(q_{1}+\cdots+q_{i}\right)-} \boldsymbol{\phi}^{i}\left(\tilde{\boldsymbol{x}}^{q}(s), \zeta\right), & \text { if } i \in \tilde{\mathcal{I}}, \\ \lim _{s \rightarrow\left(q_{1}+\cdots+q_{i}\right)-} \tilde{\boldsymbol{x}}^{q}(s), & \text { if } i \notin \tilde{\mathcal{I}},\end{cases}
$$


where

$$
\tilde{\mathcal{I}}=\left\{i \in\{1, \cdots, p-1\}: \theta_{1}^{i}+\cdots+\theta_{q_{i}}^{i}>0\right\} .
$$

Similarly, applying version 2 of the time-scaling transformation to (4.22) yields

$$
\boldsymbol{y}^{i+1,1}(0)= \begin{cases}\boldsymbol{\phi}^{i}\left(\boldsymbol{y}^{i, q_{i}}(1), \zeta\right), & \text { if } i \in \tilde{\mathcal{I}} \\ \boldsymbol{y}^{i, q_{i}}(1), & \text { if } i \notin \tilde{\mathcal{I}}\end{cases}
$$

Note that the number of jumps in (4.22) is consistent with the number of jumps in (4.23) and (4.24) (in fact, $\tilde{\mathcal{I}}=\mathcal{I}$ ). Thus, using (4.22) instead of (4.18) ensures that the time-scaling transformation maintains consistency with regards to the number of state jumps in the new and old time horizons. Imposing (4.22), however, leads to discontinuities in the switched system optimal control problem. Methods for tackling these discontinuities are described in [8, 42].

As an alternative to (4.22), one can simply assume that every stage operates for a non-negligible amount of time, thus ensuring that adjacent switching times never coincide [31]. This can be achieved by imposing the following constraints:

$$
\tau_{i}-\tau_{i-1} \geqslant \rho_{i}, \quad i=1, \cdots, p,
$$

where $\rho_{i}>0$ denotes the minimum duration of stage $i$. However, these constraints remove the flexibility to eliminate "non-optimal" subsystems by merging two or more consecutive switching times into a single switch.

\section{Variable Switching Sequence}

We are now ready to tackle the most general class of switched systems: those in which both the switching times and the switching sequence are decision variables to be optimized. The major difficulty with optimizing such systems is that the switching sequence is a discrete optimization variable, so applying the control parameterization method invariably leads to a mixed-integer programming problem rather than a nonlinear programming problem. In this section, we will discuss three popular approaches for overcoming this difficulty.

\subsection{Heuristic Approach}

Recall our prototypical switched system from the problem formulation in Sect. 2:

$$
\begin{aligned}
\dot{\boldsymbol{x}}(t) & =\boldsymbol{f}^{v_{i}}(t, \boldsymbol{x}(t), \boldsymbol{u}(t), \zeta), \quad t \in\left[\tau_{i-1}, \tau_{i}\right), i=1, \cdots, p, \\
\boldsymbol{x}\left(\tau_{i}^{+}\right) & =\boldsymbol{\phi}^{i}\left(\boldsymbol{x}\left(\tau_{i}^{-}\right), \zeta\right), \quad i=1, \cdots, p-1, \\
\boldsymbol{x}(0) & =\boldsymbol{x}^{0}
\end{aligned}
$$

This switched system evolves over $p$ stages, with the system mode fixed during each stage. Thus, there are at most $p-1$ mode switches. The problem is to determine the optimal mode for each stage and the corresponding stage duration. 
Recall that there are $m$ possible modes-i.e. $v_{i} \in\{1, \cdots, m\}$ for each $i=$ $1, \cdots, p$. To allow for every possible switching sequence, we divide each subinterval $\left[\tau_{i-1}, \tau_{i}\right), i=1, \cdots, p$, into $m$ minor subintervals-one for each element in $\{1, \cdots, m\}$. The mode on each minor subinterval is then set to the corresponding element in $\{1, \cdots, m\}$. As we will see, by introducing additional subintervals in this manner, any switching sequence with at most $p-1$ switches can be replicated by manipulating the subinterval lengths. Thus, the problem of determining an optimal switching sequence is reduced to a switching time optimization problem. We discuss the details of this transformation procedure below.

Let $\eta_{k}, k=1, \cdots, m p-1$, be new switching times that satisfy the following constraints:

$$
0=\eta_{0} \leqslant \eta_{1} \leqslant \eta_{2} \leqslant \cdots \leqslant \eta_{m p-1} \leqslant \eta_{m p}=T .
$$

These new switching times partition the time horizon into subintervals $\left[\eta_{k-1}, \eta_{k}\right)$, $k=1, \cdots, m p$.

Define

$$
\omega_{k}=(k-1) \bmod m+1, \quad k=1, \cdots, m p,
$$

where "mod" denotes the remainder. Notice that the sequence $\left\{\omega_{k}\right\}_{k=1}^{m p}$ evolves as follows:

$$
\underbrace{1,2, \cdots, m, 1,2, \cdots, m, \cdots, 1,2, \cdots, m}_{p \text { cycles }}
$$

Thus, $\left\{\omega_{k}\right\}_{k=1}^{m p}$ can be divided into $p$ cycles, where each cycle is a duplicate of the sequence $\{1, \cdots, m\}$.

Suppose that we set $\tau_{i}=\eta_{m i}, i=1, \cdots, p$, in the switched system (5.1)-(5.3). Then the partition $\left[\eta_{k-1}, \eta_{k}\right), k=1, \cdots, m p$, divides each stage $\left[\tau_{i-1}, \tau_{i}\right)$ into $m$ minor subintervals. Using $\omega_{k}$ as the system mode on the $k$ th minor subinterval, we obtain the following switched system:

$$
\begin{aligned}
\dot{\boldsymbol{x}}(t) & =\boldsymbol{f}^{\omega_{k}}(t, \boldsymbol{x}(t), \boldsymbol{u}(t), \zeta), \quad t \in\left[\eta_{k-1}, \eta_{k}\right), k=1, \cdots, m p, \\
\boldsymbol{x}\left(\eta_{m i}^{+}\right) & =\boldsymbol{\phi}^{i}\left(\boldsymbol{x}\left(\eta_{m i}^{-}\right), \zeta\right), \quad i=1, \cdots, p-1, \\
\boldsymbol{x}(0) & =\boldsymbol{x}^{0}
\end{aligned}
$$

This situation is illustrated in Fig. 4.

Recall that $\left\{\omega_{k}\right\}_{k=1}^{m p}$ consists of $p$ cycles, where each cycle is a copy of the sequence $\{1, \cdots, m\}$. Suppose that in each cycle, we select just one value from $\{1, \cdots, m\}$ and delete the other values by making the durations of the corresponding subintervals zero (a subinterval can be "deleted" by making its start and end times coincide). Then the new switched system (5.5)-(5.7) will switch mode at most $p-1$ times, just like the original switched system (5.1)-(5.3). In fact, by judiciously choosing $\eta_{k}, k=1, \cdots, m p$, we can make (5.5)-(5.7) model (5.1)-(5.3) for any switching sequence of length $p$. For example, let $m=3, p=4$, and consider the switching sequence $\{1,3,1,2\}$ in (5.1)-(5.3). This switching sequence can be replicated in (5.5)- 


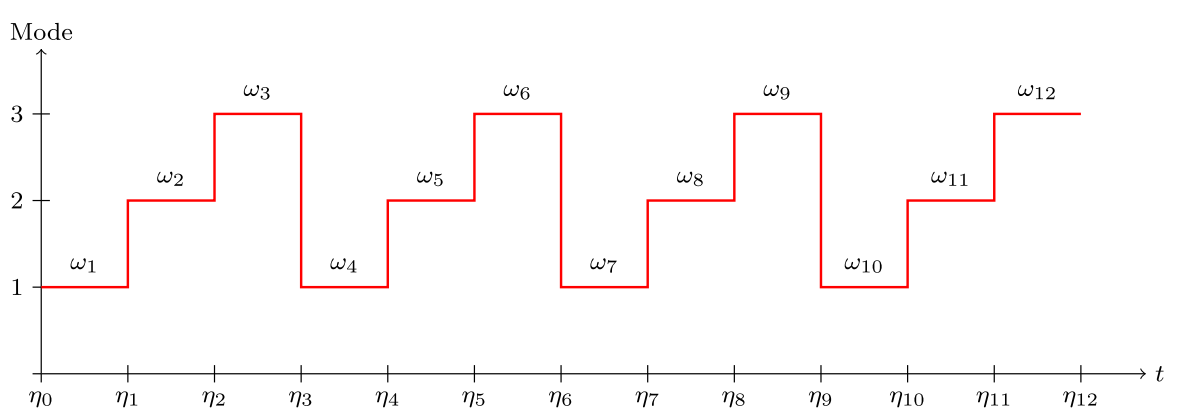

Fig. 4 Example of $\left\{\omega_{k}\right\}_{k=1}^{m p}$ for $m=3$ and $p=4$

(5.7) by choosing

$$
0<\eta_{1}=\eta_{2}=\eta_{3}=\eta_{4}=\eta_{5}<\eta_{6}<\eta_{7}=\eta_{8}=\eta_{9}=\eta_{10}<\eta_{11}=\eta_{12}=T .
$$

Here, subintervals $2,3,4,5,8,9,10$, and 12 have been deleted in (5.5) to produce the switching sequence $\{1,3,1,2\}$.

Unfortunately, the new switched system (5.5)-(5.7) is not completely equivalent to (5.1)-(5.3). This is because although (5.5)-(5.7) can be used to generate any switching sequence of length $p$ (and therefore any switching sequence for the original switched system (5.1)-(5.3)), it can also generate switching sequences of length greater than $p$. Indeed, there are potentially $m p-1$ switches in (5.5)-(5.7). In general, there is no way of ensuring that the optimal values of $\eta_{k}, k=1, \cdots, m p$, will result in a switching sequence of length $p$. Thus, using (5.5)-(5.7) to model (5.1)-(5.3) is only a heuristic approach. Nevertheless, this approach often works well in practice, especially when there are no hard restrictions on the number of mode switches allowed. See $[27,53]$ for more details.

The chief advantage with this heuristic approach is that it converts the original problem, in which the mode sequence is a discrete optimization variable, into a switching time optimization problem, which can be solved readily using the timescaling transformation described in Sect. 4. The major disadvantage is that this conversion is not an equivalent transformation, and problems may arise when the governing switched system involves state jumps (the state jump conditions in the new system are only imposed at every $m$ th switch; see (5.6)). The computational strategies discussed in the next two subsections do not suffer from these limitations.

\subsection{Bilevel Optimization Approach}

In its most general form, the switched system optimal control problem formulated in Sect. 2 involves the following decision variables:

- The control function $\boldsymbol{u}:[0, T] \rightarrow \mathbb{R}^{r}$;

- The parameter vector $\zeta \in \mathbb{R}^{w}$;

- The switching time vector $\boldsymbol{\tau}=\left[\tau_{1}, \cdots, \tau_{p-1}\right]^{\top} \in \mathbb{R}^{p-1}$; and

- The switching sequence vector $\boldsymbol{v}=\left[v_{1}, \cdots, v_{p}\right]^{\top} \in\{1, \cdots, m\} \times \cdots \times\{1, \cdots, m\}$. 
Our task is to choose these decision variables optimally to minimize the cost $g_{0}=$ $g_{0}(\boldsymbol{u}, \zeta, \boldsymbol{\tau}, \boldsymbol{v})$. Note that the control function, the parameter vector, and the switching time vector are continuous-valued decision variables, while the switching sequence is a discrete decision variable.

In the bilevel optimization approach [63, 64, 68], the switched system optimal control problem is decomposed into the following bilevel form:

$$
\min _{\boldsymbol{v}} \min _{\boldsymbol{u}, \boldsymbol{\zeta}, \boldsymbol{\tau}} g_{0}(\boldsymbol{u}, \zeta, \boldsymbol{\tau}, \boldsymbol{v})
$$

There are two levels of minimization in (5.8): in the outer level, the switching sequence is optimized; in the inner level, the switching sequence is fixed, while the control function, parameter vector, and subsystem switching times are optimized subject to the canonical constraints (2.6) and the continuous inequality constraints (2.7). Thus, the outer level of (5.8) involves solving a discrete optimization problem, while the inner level involves solving a switched system optimal control problem with fixed mode sequence.

We can rewrite (5.8) as follows:

$$
\min _{v} J(v)
$$

where

$$
J(\boldsymbol{v})=\min _{\boldsymbol{u}, \zeta, \boldsymbol{\tau}} g_{0}(\boldsymbol{u}, \zeta, \boldsymbol{\tau}, \boldsymbol{v}) .
$$

In (5.9), the switched system optimal control problem is expressed as a discrete optimization problem in which the mode sequence is the decision variable, and the value of the cost function is obtained by fixing the mode sequence and then minimizing with respect to the continuous-valued decision variables. To compute $J(\boldsymbol{v})$ for each switching sequence vector $\boldsymbol{v}$, the control parameterization and time-scaling techniques described in Sects. 3 and 4 can be applied. To minimize $J(\boldsymbol{v})$ in the outer level minimization, discrete optimization techniques, such as particle swarm optimization [13] or the filled function method [65, 74], must be applied. For more details, see $[63,64,68]$.

\subsection{Binary Relaxation Approach}

In this approach, we introduce the following binary decision variables:

$$
z_{i j}= \begin{cases}1, & \text { if mode } j \text { is active during stage } i \\ 0, & \text { otherwise }\end{cases}
$$

With these new binary decision variables, the switched system dynamics can be written as follows:

$$
\dot{\boldsymbol{x}}(t)=\sum_{j=1}^{m} z_{i j} \boldsymbol{f}^{j}(t, \boldsymbol{x}(t), \boldsymbol{u}(t), \zeta), \quad t \in\left[\tau_{i-1}, \tau_{i}\right), i=1, \cdots, p .
$$


In these new dynamics, the switching sequence is fixed; the active mode during each stage is governed by the new binary decision variables $z_{i j}, i=1, \cdots, p$, $j=1, \cdots, m$.

We now relax these binary decision variables by replacing the $0-1$ constraints with equivalent constraints in the continuous domain. More specifically, we replace $z_{i j} \in\{0,1\}, i=1, \cdots, p, j=1, \cdots, m$, with the new constraints

$$
0 \leqslant z_{i j} \leqslant 1, \quad i=1, \cdots, p, j=1, \cdots, m,
$$

and

$$
z_{i j}\left(1-z_{i j}\right) \leqslant 0, \quad i=1, \cdots, p, j=1, \cdots, m .
$$

It is clear that (5.12) and (5.13) are equivalent to $z_{i j} \in\{0,1\}, i=1, \cdots, p, j=$ $1, \cdots, m$. Thus, by imposing (5.12) and (5.13), we can view $z_{i j}$ as a continuousvalued decision variable rather than a discrete variable. This allows $z_{i j}$ to be optimized using continuous optimization techniques.

To ensure that only one subsystem is active during each stage, we impose the following additional constraints:

$$
\sum_{j=1}^{m} z_{i j}=1, \quad i=1, \cdots, p
$$

These constraints ensure that for each $i=1, \cdots, p$, there exists exactly one $j^{\prime} \in$ $\{1, \cdots, m\}$ such that $z_{i j^{\prime}}=1$ and $z_{i j}=0$ for all $j \neq j^{\prime}$. Thus, imposing (5.12)-(5.14) guarantees that $z_{i j}$ is consistent with the original definition given in (5.10). The new optimal control problem involves choosing $z_{i j}, i=1, \cdots, p, j=1, \cdots, m$, together with the original decision variables $\boldsymbol{u}, \boldsymbol{\zeta}$, and $\boldsymbol{\tau}$, to minimize the system cost subject to the new dynamics (5.11). In principle, this new problem-a switched system optimal control problem with fixed mode sequence-can be solved using the control parameterization and time-scaling techniques described in Sects. 3 and 4. Note, however, that standard gradient-based optimization methods will usually struggle with (5.13), as these constraints essentially restrict the feasible region to a disjoint set. Thus, in practice, the exact penalty method developed in [71-73] (see also Sect. 3.2) must be deployed to handle these constraints.

As an alternative to (5.13), the following equality constraints can be used:

$$
\sum_{j=1}^{m} j^{2} z_{i j}-\left\{\sum_{j=1}^{m} j z_{i j}\right\}^{2}=0, \quad i=1, \cdots, p .
$$

It can be shown (see [34, 62]) that constraints (5.12), (5.14), and (5.15) imply $z_{i j} \in$ $\{0,1\}, i=1, \cdots, p, j=1, \cdots, m$.

Another alternative to (5.13) is

$$
\sum_{j=1}^{m} z_{i j}\left(j^{2}-j+\frac{1}{3}\right)-\left\{\sum_{j=1}^{m} z_{i j}\left(j-\frac{1}{2}\right)\right\}^{2}=\frac{1}{12}, \quad i=1, \cdots, p .
$$


These equality constraints were introduced in [12] to solve a non-smooth optimal control problem arising in gradient-elution chromatography. It can be shown that imposing (5.16) together with (5.12) and (5.14) ensures that $z_{i j}$ is binary. As with (5.13), the exact penalty method is usually needed to handle constraints (5.15) and (5.16); standard optimization algorithms are generally not sufficient.

To conclude this section, we mention that there is another relaxation technique in the literature based on control integral approximations that can also be applied to handle the binary decision variables $z_{i j}$; for details, see $[16,17]$.

\section{Applications}

In Sects. 3-5, we discussed several computational strategies for solving switched system optimal control problems. We now demonstrate the real-world applicability of the switched system framework by presenting a number of practical switched system optimal control models.

\subsection{Switched-Capacitor DC/DC Power Converters}

A switched-capacitor DC/DC power converter is a small electronic device, consisting primarily of switches and capacitors, that generates different DC voltages from a single DC source. Such power converters are commonly used in mobile electronic devices such as laptop computers and cellular phones.

The circuit topology in a switched-capacitor DC/DC power converter is governed by the switch configuration. For each switch configuration, some of the capacitors act as the power supply and deliver energy to the load, while the remaining capacitors are charged by the input source. When the circuit topology is changed, the capacitors' roles are reversed: those capacitors that were previously storing energy begin to supply energy to the load, while those capacitors that were previously supplying energy to the load begin to store energy. The power converter operates by regularly switching between the different circuit topologies to produce a steady output voltage. An introduction to the operation of switched-capacitor DC/DC power converters is given in [19].

Consider a switched-capacitor DC/DC power converter with $m$ circuit topologies and $p$ operating stages. The $j$ th circuit topology can be modeled mathematically by the following linear system:

$$
\dot{\boldsymbol{x}}(t)=A^{j}\left(R_{L}\right) \boldsymbol{x}(t)+B^{j}\left(R_{L}\right) \boldsymbol{\sigma},
$$

where $\boldsymbol{x}(t) \in \mathbb{R}^{n}$ contains the capacitor voltages, $\sigma \in \mathbb{R}^{w}$ contains the DC input voltages, $R_{L} \in \mathbb{R}$ is the load resistance, and $A^{j}: \mathbb{R} \rightarrow \mathbb{R}^{n \times n}$ and $B^{j}: \mathbb{R} \rightarrow \mathbb{R}^{n \times w}$ are given matrix-valued functions of the load resistance (these functions can be derived using Kirchhoff's circuit laws).

The output voltage delivered by the power converter during the $j$ th circuit topology is given by

$$
y(t)=C^{j}\left(R_{L}\right) x(t)+D^{j}\left(R_{L}\right) \sigma,
$$


where $C^{j}: \mathbb{R} \rightarrow \mathbb{R}^{1 \times n}$ and $D^{j}: \mathbb{R} \rightarrow \mathbb{R}^{1 \times w}$ are given matrix-valued functions of the load resistance.

Let $\tau_{i}, i=1, \cdots, p-1$, denote the topology switching times, with $\tau_{0}=0$ and $\tau_{p}=T$. Then the operation of the switched-capacitor DC/DC power converter can be described by the following switched system:

$$
\left.\begin{array}{l}
\dot{\boldsymbol{x}}(t)=A^{v_{i}}\left(R_{L}\right) \boldsymbol{x}(t)+B^{v_{i}}\left(R_{L}\right) \boldsymbol{\sigma} \\
y(t)=C^{v_{i}}\left(R_{L}\right) \boldsymbol{x}(t)+D^{v_{i}}\left(R_{L}\right) \boldsymbol{\sigma}
\end{array}\right\} \quad t \in\left[\tau_{i-1}, \tau_{i}\right), i=1, \cdots, p,
$$

where $v_{i} \in\{1, \cdots, m\}$ denotes the active topology during the $i$ th stage. The initial conditions are

$$
\boldsymbol{x}(0)=x^{0}
$$

where $\boldsymbol{x}^{0} \in \mathbb{R}^{n}$ is a given vector. Furthermore, because topology switches are accompanied by instantaneous voltage leaks, there is a state jump occurring at each switching time:

$$
\boldsymbol{x}\left(\tau_{i}^{+}\right)=\boldsymbol{x}\left(\tau_{i}^{-}\right)+\boldsymbol{\phi}^{i}\left(\boldsymbol{x}\left(\tau_{i}^{-}\right)\right), \quad i=1, \cdots, p-1,
$$

where $\boldsymbol{\phi}^{i}: \mathbb{R}^{n} \rightarrow \mathbb{R}^{n}, i=1, \cdots, p-1$, are given functions describing the magnitudes of the voltage leaks. A method for determining these functions is described in [18].

When designing a control policy for a switched-capacitor DC/DC power converter, the following quantities should be considered:

(1) Output voltage ripple (the power converter should deliver a steady voltage to the attached load);

(2) Output voltage sensitivity with respect to the load resistance (changing the attached load should not cause large changes in the output voltage); and

(3) Output voltage sensitivity with respect to the input voltage (random fluctuations in the input voltage should not cause large changes in the output voltage).

Based on these criteria, we define the following cost function:

$$
g_{0}=\alpha_{1}\{\underbrace{\sup _{t \in[0, T]} y(t)-\inf _{t \in[0, T]} y(t)}_{\text {Output voltage ripple }}\}+\alpha_{2} \underbrace{\sup _{t \in[0, T]}\left|\frac{\partial y(t)}{\partial R_{L}}\right|}_{\text {Output sensitivity }}+\alpha_{3} \underbrace{\sup _{t \in[0, T]}\left\|\frac{\partial y(t)}{\partial \boldsymbol{\sigma}}\right\|}_{\text {Output sensitivity }}
$$

where $\alpha_{1}, \alpha_{2}$, and $\alpha_{3}$ are given weights and $\|\cdot\|$ denotes an appropriate norm.

The problem is to minimize (6.4) subject to the switched system (6.1)-(6.3). The decision variables in this problem are the switching times and the switching sequence. See $[18,44]$ for more details, including optimization algorithms based on the timescaling transformation.

\subsection{Aquaculture Operations}

Aquaculture farming refers to the process of rearing seafood (e.g. tuna, mussels, salmon, crustaceans) for human consumption. In this section, we consider the aquaculture farming of shrimp. The aim is to determine the optimal times at which to 
harvest shrimp from an aquaculture pond so that total revenue from the aquaculture operation is maximized. As we will see, this problem can be formulated as an impulsive optimal control problem.

Let $x_{1}(t)$ denote the number of shrimp in the pond at time $t$. Furthermore, let $x_{2}(t)$ denote the average weight of shrimp (in grams) at time $t$. Then according to [70], the state variables $x_{1}$ and $x_{2}$ can be modeled by the following differential equations:

$$
\begin{aligned}
& \dot{x}_{1}(t)=-\alpha_{1} x_{1}(t), \\
& \dot{x}_{2}(t)=\alpha_{2}-\alpha_{3} x_{1}(t) x_{2}(t),
\end{aligned}
$$

where $\alpha_{1}, \alpha_{2}$, and $\alpha_{3}$ are model constants. Equation (6.5) models shrimp mortality by an exponential decay process, while (6.6) expresses the shrimp growth rate as a function of the biomass in the pond (the more shrimp in the pond, the more competition for food).

The initial conditions for the state variables are

$$
x_{1}(0)=x_{1}^{0}, \quad x_{2}(0)=x_{2}^{0},
$$

where $x_{1}^{0}>0$ and $x_{2}^{0}>0$ are given constants.

Let $\tau_{i}$ denote the $i$ th harvest time. Furthermore, let $\zeta_{i}$ denote the fraction of shrimp stock extracted at the $i$ th harvest. Then

$$
x_{1}\left(\tau_{i}^{+}\right)=x_{1}\left(\tau_{i}^{-}\right)-\zeta_{i} x_{1}\left(\tau_{i}^{-}\right), \quad i=1, \cdots, p,
$$

and

$$
x_{2}\left(\tau_{i}^{+}\right)=x_{2}\left(\tau_{i}^{-}\right), \quad i=1, \cdots, p .
$$

Equation (6.8) is a state jump condition: it indicates that the shrimp population decreases instantaneously at each harvest time when shrimp are removed from the pond. Equation (6.9) simply states that the average weight of shrimp is unchanged by the $i$ th harvest.

The decision variables $\tau_{i}, i=1, \cdots, p$, and $\zeta_{i}, i=1, \cdots, p$, satisfy the following constraints:

$$
0 \leqslant \tau_{1} \leqslant \tau_{2} \leqslant \cdots \leqslant \tau_{p}
$$

and

$$
0 \leqslant \zeta_{i} \leqslant 1, \quad i=1, \cdots, p .
$$

The revenue obtained over the production cycle $\left[0, \tau_{p}\right]$ is given by

$$
g_{0}=\sum_{i=1}^{p}\left\{\beta \zeta_{i} x_{1}\left(\tau_{i}^{-}\right) x_{2}\left(\tau_{i}^{-}\right)-h\right\},
$$

where $\beta$ is the price of shrimp (in dollars per gram) and $h$ is the fixed cost of a single harvest (in dollars). The problem is to choose the harvest times $\tau_{i}, i=1, \cdots, p$, and the harvest fractions $\zeta_{i}, i=1, \cdots, p$, to maximize the total revenue (6.12) subject to the dynamic system (6.5)-(6.9) and the constraints (6.10) and (6.11). 
Technically speaking, since its dynamics do not change at the harvest times, system (6.5)-(6.9) is an impulsive system rather than a switched system. Nevertheless, such impulsive systems can be viewed as special cases of the general switched system formulation given in Sect. 2.

The above optimal control problem was solved using the time-scaling transformation in references [31, 70]. Later, in references [22, 23], a neighboring extremal approach was developed to determine an optimal feedback control policy. A variation of this optimal control problem in which the price of shrimp is defined in terms of a piecewise-constant function of the average shrimp weight is considered in reference [7].

\subsection{1,3-Propanediol Production}

1,3-Propanediol is an organic compound widely used in the production of industrial polymers. In this section, we consider a fed-batch fermentation process for manufacturing 1,3-propanediol via glycerol bio-dissimilation. This process consists of two modes: feeding mode, during which glycerol is added continuously to the fermentor, and batch mode, during which the glycerol feed is stopped. The fed-batch process switches back and forth between the batch and feeding modes to maintain a suitable environment for cell growth.

We consider the fed-batch model described in reference [35]. The state vector in this model is $\boldsymbol{x}(t)=\left[x_{1}(t), x_{2}(t), x_{3}(t), x_{4}(t), x_{5}(t)\right]^{\top}$, where:

- $x_{1}(t)$ is the concentration of biomass at time $t$;

- $x_{2}(t)$ is the concentration of glycerol at time $t$;

- $x_{3}(t)$ is the concentration of 1,3-propanediol at time $t$;

- $x_{4}(t)$ is the concentration of acetic acid at time $t$; and

- $x_{5}(t)$ is the concentration of ethanol at time $t$.

In batch mode, the mass balance equations are

$$
\begin{aligned}
& \dot{x}_{1}(t)=\beta_{1}(x(t)) x_{1}(t), \\
& \dot{x}_{2}(t)=-\beta_{2}(x(t)) x_{1}(t), \\
& \dot{x}_{3}(t)=\beta_{3}(x(t)) x_{1}(t), \\
& \dot{x}_{4}(t)=\beta_{4}(x(t)) x_{1}(t), \\
& \dot{x}_{5}(t)=\beta_{5}(x(t)) x_{1}(t),
\end{aligned}
$$

where

$$
\begin{aligned}
& \beta_{1}(\boldsymbol{x}(t))=\frac{\Delta_{1} x_{2}(t)}{x_{2}(t)+\alpha_{1}} \prod_{l=2}^{5}\left\{1-\frac{x_{l}(t)}{x_{l}^{*}}\right\}^{\kappa_{l}}, \\
& \beta_{l}(\boldsymbol{x}(t))=v_{l}+z_{l} \beta_{1}(\boldsymbol{x}(t))+\frac{\Delta_{l} x_{2}(t)}{x_{2}(t)+\alpha_{l}}, \quad l=2,3,4, \\
& \beta_{5}(\boldsymbol{x}(t))=\beta_{2}(\boldsymbol{x}(t))\left\{\frac{c_{1}}{c_{2}+\beta_{1}(\boldsymbol{x}(t)) x_{2}(t)}+\frac{c_{3}}{c_{4}+\beta_{1}(\boldsymbol{x}(t)) x_{2}(t)}\right\} .
\end{aligned}
$$


Here, $\kappa_{2}=1$ and $\kappa_{3}=\kappa_{4}=\kappa_{5}=3$, while $\Delta_{l}, \alpha_{l}, x_{l}^{*}, v_{l}, z_{l}$, and $c_{1}, c_{2}, c_{3}$, and $c_{4}$ are model constants. The problem of determining optimal estimates for these constants is considered in [38].

In feeding mode, the mass balance equations are

$$
\begin{aligned}
& \dot{x}_{1}(t)=\beta_{1}(\boldsymbol{x}(t)) x_{1}(t)-D(t, u(t)) x_{1}(t), \\
& \dot{x}_{2}(t)=D(t, u(t))\left\{\frac{c_{0}}{1+\rho}-x_{2}(t)\right\}-\beta_{2}(\boldsymbol{x}(t)) x_{1}(t), \\
& \dot{x}_{3}(t)=\beta_{3}(\boldsymbol{x}(t)) x_{1}(t)-D(t, u(t)) x_{3}(t), \\
& \dot{x}_{4}(t)=\beta_{4}(\boldsymbol{x}(t)) x_{1}(t)-D(t, u(t)) x_{4}(t), \\
& \dot{x}_{5}(t)=\beta_{5}(\boldsymbol{x}(t)) x_{1}(t)-D(t, u(t)) x_{5}(t),
\end{aligned}
$$

where $u(t)$ is the glycerol feeding rate at time $t, \rho$ is the ratio of the feed velocity of alkali to the feed velocity of glycerol, $c_{0}$ is the initial concentration of glycerol feed, and

$$
\begin{aligned}
D(t, u(t)) & =\frac{(1+\rho) u(t)}{V(t)}, \\
V(t) & =V_{0}+\int_{0}^{t}(1+\rho) u(s) d s .
\end{aligned}
$$

Because of physical limitations, the rate at which glycerol is added to the fermentor during feeding mode is subject to the following bound constraints:

$$
a \leqslant u(t) \leqslant b, \quad t \in[0, T]
$$

where $T$ is the terminal time and $a$ and $b$ are given constants such that $0<a<b$.

The problem is to maximize $x_{3}(T)$, the yield of 1,3-propanediol at the terminal time, subject to the batch and feeding mode dynamics. The decision variables in this problem are the times at which the system switches between batch and feeding modes (i.e. the times at which the system dynamics switch between (6.13)-(6.17) and (6.18)-(6.22)), as well as the feeding rate of glycerol during the feeding mode. See $[35,37]$ for more details.

\subsection{Diesel-Electric Submarines}

We consider the dynamic model of a diesel-electric submarine presented in [50]. The submarine has a battery-powered propulsion system and three diesel generators for recharging the battery. Each generator has two operating modes: normal mode and supercharged mode. Supercharged mode gives a faster recharge rate, but creates more noise. The aim is to find an optimal strategy for running the generators so that adequate charge in the battery is maintained, the submarine travels a required distance, and the noise generated by the submarine is minimized. 
Table 1 The possible operating modes for the submarine described in Sect. 6.4. Here,

$u_{\text {noise }}$ is a given constant representing the minimum noise level

\begin{tabular}{lll}
\hline Operating Mode & $u_{1}$ & $u_{2}$ \\
\hline Discharging (generators off) & 0 & 0 \\
Recharging (1 normal) & 0.2564 & $u_{\text {noise }}$ \\
Recharging (1 supercharged) & 0.3333 & $1.5 \times u_{\text {noise }}$ \\
Recharging (2 normal) & 0.5128 & $2.0 \times u_{\text {noise }}$ \\
Recharging (2 supercharged) & 0.6666 & $3.0 \times u_{\text {noise }}$ \\
Recharging (3 normal) & 0.7692 & $3.0 \times u_{\text {noise }}$ \\
Recharging (3 supercharged) & 1.0 & $4.5 \times u_{\text {noise }}$ \\
\hline
\end{tabular}

The charge state of the submarine's battery is described by the following differential equation:

$$
\begin{aligned}
& \dot{x}(t)=R\left(x(t), u_{1}(t)\right)-D\left(u_{1}(t), u_{3}(t)\right), \quad t \in[0, T], \\
& x(0)=x_{0},
\end{aligned}
$$

where $x(t)$ is the charge state of the battery at time $t, u_{1}(t)$ is the fraction of full mechanical power produced by the generators at time $t, u_{2}(t)$ is the generator noise level at time $t, u_{3}(t)$ is the speed of the submarine at time $t, R: \mathbb{R} \times \mathbb{R} \rightarrow \mathbb{R}$ is the recharge rate, $D: \mathbb{R} \times \mathbb{R} \rightarrow \mathbb{R}$ is the discharge rate, $T$ is the journey time, and $x_{0}$ is the initial charge.

The battery recharge rate depends on the charge state of the battery and the amount of power being produced by the generators:

$$
R\left(x(t), u_{1}(t)\right)=\left(2.0 \times 10^{4}\right) \frac{u_{1}(t)}{x(t)+100} .
$$

The battery discharge rate depends primarily on the speed of the submarine:

$$
D\left(u_{1}(t), u_{3}(t)\right)= \begin{cases}\frac{1}{120} u_{3}^{2}(t)+8, & \text { if } u_{1}(t) \neq 0, \\ \frac{1}{80} u_{3}^{2}(t)+5, & \text { if } u_{1}(t)=0 .\end{cases}
$$

Controls $u_{1}:[0, T] \rightarrow \mathbb{R}$ and $u_{2}:[0, T] \rightarrow \mathbb{R}$ govern the operating mode of the submarine. There are seven modes in total, the details of which are given in Table 1.

Note that $u_{1}$ and $u_{2}$ are discrete-valued control variables that assume values in a discrete set:

$$
\begin{gathered}
{\left[\begin{array}{l}
u_{1}(t) \\
u_{2}(t)
\end{array}\right] \in\left\{\left[\begin{array}{l}
0 \\
0
\end{array}\right],\left[\begin{array}{c}
0.2564 \\
u_{\text {noise }}
\end{array}\right],\left[\begin{array}{c}
0.3333 \\
1.5 u_{\text {noise }}
\end{array}\right],\left[\begin{array}{l}
0.5128 \\
2 u_{\text {noise }}
\end{array}\right],\left[\begin{array}{l}
0.6666 \\
3 u_{\text {noise }}
\end{array}\right],\right.} \\
\left.\left[\begin{array}{l}
0.7692 \\
3 u_{\text {noise }}
\end{array}\right],\left[\begin{array}{c}
1 \\
4.5 u_{\text {noise }}
\end{array}\right]\right\} .
\end{gathered}
$$

Thus, system (6.23)-(6.24) can be viewed as a switched system in which each discrete control vector on the right-hand side of (6.25) corresponds to a different system mode. 
Physical constraints on the submarine's propulsion system mean that the submarine's speed is limited to between $5 \mathrm{~km} / \mathrm{h}$ and $60 \mathrm{~km} / \mathrm{h}$ :

$$
5 \leqslant u_{3}(t) \leqslant 60, \quad t \in[0, T] .
$$

Moreover, sufficient battery charge must be maintained throughout the journey:

$$
x_{\min } \leqslant x(t) \leqslant x_{\max }, \quad t \in[0, T],
$$

where $x_{\min }$ and $x_{\max }$ are given constants. To ensure the submarine travels a required distance of $L$ kilometers, we impose the following canonical equality constraint:

$$
\int_{0}^{T} u_{3}(t) d t=L
$$

Given weights $\alpha_{1}>0$ and $\alpha_{2}>0$, the optimal control problem is to choose $u_{1}, u_{2}$, and $u_{3}$ to minimize the cost function

$$
g_{0}=\underbrace{\alpha_{1} \int_{0}^{T}(x(t)-100)^{2} d t}_{\text {Deviation from full charge }}+\underbrace{\alpha_{2} \int_{0}^{T} \ln \left(u_{2}(t)+1\right) d t}_{\text {Noise level }}
$$

subject to the dynamic system (6.23)-(6.24) and the constraints (6.25)-(6.28). This optimal discrete-valued control problem can be formulated as a switched system optimal control problem; see Sect. 2 for details. Solution algorithms for solving this problem based on the time-scaling transformation are described in $[11,50]$.

\subsection{Sensor Scheduling}

Consider a process described by the following system of stochastic differential equations:

$$
\begin{aligned}
d \boldsymbol{x}(t) & =A(t) \boldsymbol{x}(t) d t+B(t) d \boldsymbol{z}(t), \quad t \in[0, T], \\
\boldsymbol{x}(0) & =\boldsymbol{x}^{0},
\end{aligned}
$$

where $\boldsymbol{x}(t) \in \mathbb{R}^{n}$ is the state of the process at time $t ; \boldsymbol{x}^{0} \in \mathbb{R}^{n}$ is a Gaussian random vector with mean $\overline{\boldsymbol{x}}^{0} \in \mathbb{R}^{n}$ and covariance matrix $P_{0} \in \mathbb{R}^{n \times n}$; the process $\{z(t), t \in$ $[0, T]\}$ is an $\mathbb{R}^{s}$-valued Brownian motion with mean zero and covariance matrix $Q$; and $A:[0, T] \rightarrow \mathbb{R}^{n \times n}$ and $B:[0, T] \rightarrow \mathbb{R}^{n \times s}$ are given matrix-valued functions.

We suppose that there are $m$ sensors available for making observations of the state $\boldsymbol{x}(t)$. These observations are corrupted by noise. Our goal is to use the noisy observations to construct an optimal estimate of $\boldsymbol{x}(t)$.

Only one of the $m$ sensors can be active at any one time. Let $u:[0, T] \rightarrow$ $\{1, \cdots, m\}$ be such that $u(t)=j$ if sensor $j$ is active at time $t$. This function is called the sensor schedule. 
The observation process for (6.29)-(6.30) satisfies the following system of stochastic differential equations:

$$
\begin{aligned}
d \boldsymbol{y}(t) & =\sum_{i=1}^{m}\left\{C_{i}(t) \boldsymbol{x}(t) d t+D_{i}(t) d \boldsymbol{w}_{i}(t)\right\} \chi_{\{i\}}(u(t)), \quad t \in[0, T], \\
\boldsymbol{y}(0) & =\mathbf{0}
\end{aligned}
$$

where $\boldsymbol{y}(t) \in \mathbb{R}^{d}$ is the observed process at time $t$; each $\left\{\boldsymbol{w}_{i}(t), t \in[0, T]\right\}$ is an $\mathbb{R}^{k}$ valued Brownian motion with mean zero and covariance matrix $R_{i} ; C_{i}:[0, T] \rightarrow$ $\mathbb{R}^{d \times n}$ and $D_{i}:[0, T] \rightarrow \mathbb{R}^{d \times k}$ are given matrix-valued functions; and

$$
\chi_{\{i\}}(u(t))= \begin{cases}1, & \text { if } u(t)=i \\ 0, & \text { otherwise }\end{cases}
$$

Now, let

$$
\tilde{R}_{i}^{-1}(t)=\left[D_{i}(t) R_{i} D_{i}(t)^{\top}\right]^{-1} .
$$

Our aim is to use $\boldsymbol{y}(t)$ to form an estimate of $\boldsymbol{x}(t)$. Under suitable assumptions (see $[2,5])$, it can be shown that the optimal mean-square estimate of $\boldsymbol{x}(t)$ is $\hat{\boldsymbol{x}}(t)$, where

$$
\begin{aligned}
d \hat{\boldsymbol{x}}(t)= & \left\{A(t)-P(t) \sum_{i=1}^{m} C_{i}(t)^{\top} \tilde{R}_{i}^{-1}(t) C_{i}(t) \chi_{\{i\}}(u(t))\right\} \hat{\boldsymbol{x}}(t) d t \\
& +\left\{P(t) \sum_{i=1}^{m} C_{i}(t)^{\top} \tilde{R}_{i}^{-1}(t) \chi_{\{i\}}(u(t))\right\} d \boldsymbol{y}(t), \\
\hat{\boldsymbol{x}}(0)= & \overline{\boldsymbol{x}}^{0},
\end{aligned}
$$

and the error covariance matrix $P(t)$ satisfies the following Riccati differential equation:

$$
\begin{aligned}
\dot{P}(t)= & A(t) P(t)+P(t) A(t)^{\top}+B(t) Q B(t)^{\top} \\
& -\sum_{i=1}^{m} P(t) C_{i}(t)^{\top} \tilde{R}_{i}^{-1}(t) C_{i}(t) P(t) \chi_{\{i\}}(u(t)), \\
P(0)= & P_{0} .
\end{aligned}
$$

The estimation accuracy can be improved by choosing a sensor schedule that makes the error covariance matrix small. This motivates the following optimal control problem: Choose the sensor schedule $u:[0, T] \rightarrow\{1, \cdots, m\}$ to minimize the cost function

$$
g_{0}=\operatorname{trace}\{P(T)\}+\int_{0}^{T} \operatorname{trace}\{P(t)\} d t
$$

subject to the dynamic system (6.31)-(6.32). This is an optimal discrete-valued control problem. As mentioned previously, such problems are special cases of the general 
switched system optimal control model described in Sect. 2. See [26, 63] for more details.

\section{Concluding Remarks}

This paper has reviewed the latest computational methods based on control parameterization and the time-scaling transformation for solving switched system optimal control problems with nonlinear subsystem dynamics and nonlinear state constraints. We have considered three types of switched system: those in which both the mode sequence and the switching times are fixed; those in which the mode sequence is fixed, but the switching times are input variables; and those in which both the mode sequence and the switching times are input variables. These three switched system models are sufficiently broad to encapsulate many of the real-world switched systems arising in practice.

There is, however, another important type of switched system that is not discussed in this paper - the so-called internally forced switched system in which the switching mechanism is governed by a set of switching conditions [69]. These switching conditions are usually expressed in terms of equations involving the state variables; the system switches from one mode to another when these equations are satisfied. Consequently, the switching times in an internally forced switched system are implicit functions of the control and parameter vectors-changing these vectors changes the state trajectory, which in turn changes the times at which the switching conditions are satisfied. This switching mechanism is similar to the termination mechanisms studied in $[21,30,32]$, which are governed by state-dependent stopping criteria.

Internally forced switched systems arise in many applications, including robots [9], subway train control [55], cancer chemotherapy [47], bioreactors [36], and voicecoil motors [14]. Reference [25] describes a computational method, based on the time-scaling transformation, for generating the state trajectory of an internally forced switched system. However, this method is only for solving the system, not optimizing it. Strictly speaking, the standard control parameterization and time-scaling techniques are not applicable to optimal control problems governed by internally forced switched systems, as the dynamics in such systems depend discontinuously on the system state. Nevertheless, many researchers, including the authors of this paper, have ignored this restriction and applied control parameterization and the time-scaling transformation directly to optimal control problems subject to internally forced switched systems; see [41, 47, 64] for example. An interesting avenue for future research would be to study the ramifications of this (potentially invalid) approach. New control parameterization and time-scaling techniques should be developed to specifically cater for internally forced switched systems.

\section{References}

[1] Ahmed, N.U.: Elements of Finite-Dimensional Systems and Control Theory. Longman Scientific and Technical, Essex (1988) 
[2] Ahmed, N.U.: Linear and Nonlinear Filtering for Scientists and Engineers. World Scientific, Singapore (1998)

[3] Ahmed, N.U.: Dynamic Systems and Control with Applications. World Scientific, Singapore (2006)

[4] An, Y., Xu, C., Lin, Q., Loxton, R., Teo, K.L.: Path planning for underactuated Dubins micro-robots using switching control. In: Proceedings of the 10th IEEE International Conference on Control and Automation, Hangzhou, China (2013)

[5] Anderson, B.D.O., Moore, J.B.: Optimal Control: Linear Quadratic Methods, Dover Edition. Dover Publications, New York (2007)

[6] Bengea, S.C., DeCarlo, R.A.: Optimal control of switching systems. Automatica 41, 11-27 (2005)

[7] Blanchard, E.A., Loxton, R., Rehbock, V.: A computational algorithm for a class of non-smooth optimal control problems arising in aquaculture operations. Appl. Math. Comput. 219, 8738-8746 (2013)

[8] Blanchard, E.A., Loxton, R., Rehbock, V.: Optimal control of impulsive switched systems with minimum subsystem durations. Submitted

[9] Boccadoro, M., Wardi, Y., Egerstedt, M., Verriest, E.: Optimal control of switching surfaces in hybrid dynamical systems. Discrete Event Dyn. Syst. Theory Appl. 15, 433-448 (2005)

[10] Branicky, M.S., Borkar, V.S., Mitter, S.K.: A unified framework for hybrid control: model and optimal control theory. IEEE Trans. Autom. Control 43, 31-45 (1998)

[11] Caccetta, L., Loosen, I., Rehbock, V.: Computational aspects of the optimal transit path problem. J. Ind. Manag. Optim. 4, 95-105 (2008)

[12] Chai, Q., Loxton, R., Teo, K.L., Yang, C.: A max-min control problem arising in gradient elution chromatography. Ind. Eng. Chem. Res. 51, 6137-6144 (2012)

[13] Chen, W.N., Zhang, J., Chung, H.S.H., Zhong, W.L., Wu, W.G., Shi, Y.H.: A novel set-based particle swarm optimization method for discrete optimization problems. IEEE Trans. Evol. Comput. 14, 278300 (2010)

[14] Christiansen, B., Maurer, H., Zirn, O.: Optimal control of a voice-coil-motor with Coulombic friction. In: Proceedings of the 47th IEEE Conference on Decision and Control, Cancun, Mexico (2008)

[15] Gerdts, M.: A variable time transformation method for mixed-integer optimal control problems. Optim. Control Appl. Methods 27, 169-182 (2006)

[16] Gerdts, M., Sager, S.: Mixed-integer DAE optimal control problems: necessary conditions and bounds. In: Control and Optimization with Differential-Algebraic Constraints. Society for Industrial and Applied Mathematics, Philadelphia (2012)

[17] Hante, F.M., Sager, S.: Relaxation methods for mixed-integer optimal control of partial differential equations. Comput. Optim. Appl. 55, 197-225 (2013)

[18] Ho, C.Y.F., Ling, B.W.K., Liu, Y.Q., Tam, P.K.S., Teo, K.L.: Optimal PWM control of switchedcapacitor DC-DC power converters via model transformation and enhancing control techniques. IEEE Trans. Circuits Syst. I, Regul. Pap. 55, 1382-1391 (2008)

[19] Ioinovici, A.: Switched-capacitor power electronics circuits. IEEE Circuits Syst. Mag. 1, 37-42 (2001)

[20] Jennings, L.S., Teo, K.L.: A computational algorithm for functional inequality constrained optimization problems. Automatica 26, 371-375 (1990)

[21] Jiang, C., Lin, Q., Yu, C., Teo, K.L., Duan, G.R.: An exact penalty method for free terminal time optimal control problem with continuous inequality constraints. J. Optim. Theory Appl. 154, 30-53 (2012)

[22] Jiang, C., Teo, K.L., Loxton, R., Duan, G.R.: A neighboring extremal solution for an optimal switched impulsive control problem. J. Ind. Manag. Optim. 8, 591-609 (2012)

[23] Jiang, C., Teo, K.L., Loxton, R., Duan, G.R.: A neighboring extremal solution for optimal switched impulsive control problems with large perturbations. Int. J. Innov. Comput. Inf. Control 8, 6235-6257 (2012)

[24] Kamgarpour, M., Tomlin, C.: On optimal control of non-autonomous switched systems with a fixed mode sequence. Automatica 48, 1177-1181 (2012)

[25] Lee, H.W.J., Teo, K.L.: Control parametrization enhancing technique for solving a special ODE class with state dependent switch. J. Optim. Theory Appl. 118, 55-66 (2003)

[26] Lee, H.W.J., Teo, K.L., Lim, A.E.B.: Sensor scheduling in continuous time. Automatica 37, 20172023 (2001)

[27] Lee, H.W.J., Teo, K.L., Rehbock, V., Jennings, L.S.: Control parametrization enhancing technique for optimal discrete-valued control problems. Automatica 35, 1401-1407 (1999)

[28] Li, R., Teo, K.L., Wong, K.H., Duan, G.R.: Control parameterization enhancing transform for optimal control of switched systems. Math. Comput. Model. 43, 1393-1403 (2006) 
[29] Lin, Q., Loxton, R., Teo, K.L.: The control parameterization method for nonlinear optimal control: a survey. J. Ind. Manag. Optim. Accepted

[30] Lin, Q., Loxton, R., Teo, K.L., Wu, Y.H.: A new computational method for a class of free terminal time optimal control problems. Pac. J. Optim. 7, 63-81 (2011)

[31] Lin, Q., Loxton, R., Teo, K.L., Wu, Y.H.: A new computational method for optimizing nonlinear impulsive systems. Dyn. Contin. Discrete Impuls. Syst. Ser. B, Appl. Algorithms 18, 59-76 (2011)

[32] Lin, Q., Loxton, R., Teo, K.L., Wu, Y.H.: Optimal control computation for nonlinear systems with state-dependent stopping criteria. Automatica 48, 2116-2129 (2012)

[33] Lin, Q., Loxton, R., Teo, K.L., Wu, Y.H., Yu, C.: A new exact penalty method for semi-infinite programming problems. J. Comput. Appl. Math. Accepted subject to minor changes

[34] Liu, Y., Eberhard, A., Teo, K.L.: A numerical method for a class of mixed switching and impulsive optimal control problems. Comput. Math. Appl. 52, 625-636 (2006)

[35] Liu, C., Gong, Z., Feng, E., Yin, H.: Modelling and optimal control for nonlinear multistage dynamical system of microbial fed-batch culture. J. Ind. Manag. Optim. 5, 835-850 (2009)

[36] Liu, C., Gong, Z., Feng, E., Yin, H.: Optimal switching control of a fed-batch fermentation process. J. Glob. Optim. 52, 265-280 (2012)

[37] Liu, C., Gong, Z., Shen, B., Feng, E.: Modelling and optimal control for a fed-batch fermentation process. Appl. Math. Model. 37, 695-706 (2013)

[38] Liu, C., Loxton, R., Teo, K.L.: Optimal parameter selection for nonlinear multistage systems with time-delays. Submitted

[39] Liu, Y., Teo, K.L., Jennings, L.S., Wang, S.: On a class of optimal control problems with state jumps. J. Optim. Theory Appl. 98, 65-82 (1998)

[40] Loxton, R., Lin, Q., Rehbock, V., Teo, K.L.: Control parameterization for optimal control problems with continuous inequality constraints: new convergence results. Numer. Algebra Control Optim. 2, 571-599 (2012)

[41] Loxton, R., Teo, K.L., Rehbock, V.: Optimal control problems with multiple characteristic time points in the objective and constraints. Automatica 44, 2923-2929 (2008)

[42] Loxton, R., Teo, K.L., Rehbock, V.: Computational method for a class of switched system optimal control problems. IEEE Trans. Autom. Control 54, 2455-2460 (2009)

[43] Loxton, R., Teo, K.L., Rehbock, V.: Robust suboptimal control of nonlinear systems. Appl. Math. Comput. 217, 6566-6576 (2011)

[44] Loxton, R., Teo, K.L., Rehbock, V., Ling, W.K.: Optimal switching instants for a switched-capacitor DC/DC power converter. Automatica 45, 973-980 (2009)

[45] Loxton, R., Teo, K.L., Rehbock, V., Yiu, K.F.C.: Optimal control problems with a continuous inequality constraint on the state and the control. Automatica 45, 2250-2257 (2009)

[46] Luenberger, D.G., Ye, Y.: Linear and Nonlinear Programming, 3rd edn. Springer, New York (2008)

[47] Martin, R.B.: Optimal control drug scheduling of cancer chemotherapy. Automatica 28, 1113-1123 (1992)

[48] Nocedal, J., Wright, S.J.: Numerical Optimization, 2nd edn. Springer, New York (2006)

[49] Pontryagin, L.S., Boltyanskii, V.G., Gamkrelidze, R.V., Mishchenko, E.F.: The Mathematical Theory of Optimal Processes, L.S. Pontryagin Selected Works, vol. 4. Gordon and Breach Science Publishers, Montreux (1986)

[50] Rehbock, V., Caccetta, L.: Two defence applications involving discrete valued optimal control. ANZIAM J. 44, E33-E54 (2002)

[51] Ridenour, J., Hu, J., Pettis, N., Lu, Y.H.: Low-power buffer management for streaming data. IEEE Trans. Circuits Syst. Video Technol. 17, 143-157 (2007)

[52] Rinehart, M., Dahleh, M., Reed, D., Kolmanovsky, I.: Suboptimal control of switched systems with an application to the DISC engine. IEEE Trans. Control Syst. Technol. 16, 189-201 (2008)

[53] Ruby, T., Rehbock, V.: Numerical solutions of optimal switching control problems. In: Optimization and Control with Applications. Springer, New York (2005)

[54] Ruby, T., Rehbock, V., Lawrance, W.B.: Optimal control of hybrid power systems. Dyn. Contin. Discrete Impuls. Syst. Ser. B, Appl. Algorithms 10, 429-439 (2003)

[55] Sager, S., Bock, H.G., Reinelt, G.: Direct methods with maximal lower bound for mixed-integer optimal control problems. Math. Program., Ser. A 118, 109-149 (2009)

[56] Siburian, A., Rehbock, V.: Numerical procedure for solving a class of singular optimal control problems. Optim. Methods Softw. 19, 413-426 (2004)

[57] Stewart, D.E.: A numerical algorithm for optimal control problems with switching costs. J. Aust. Math. Soc. Ser. B, Appl. Math 34, 212-228 (1992) 
[58] Sussmann, H.J.: A maximum principle for hybrid optimal control problems. In: Proceedings of the 38th IEEE Conference on Decision and Control, Phoenix, USA (1999)

[59] Teo, K.L., Goh, C.J., Wong, K.H.: A Unified Computational Approach to Optimal Control Problems. Longman Scientific and Technical, Essex (1991)

[60] Teo, K.L., Rehbock, V., Jennings, L.S.: A new computational algorithm for functional inequality constrained optimization problems. Automatica 29, 789-792 (1993)

[61] Vincent, T.L., Grantham, W.J.: Optimality in Parametric Systems. Wiley, New York (1981)

[62] Wang, S., Teo, K.L., Lee, H.W.J.: A new approach to nonlinear mixed discrete programming problems. Eng. Optim. 30, 249-262 (1998)

[63] Woon, S.F., Rehbock, V., Loxton, R.: Global optimization method for continuous-time sensor scheduling. Nonlinear Dyn. Syst. Theory 10, 175-188 (2010)

[64] Woon, S.F., Rehbock, V., Loxton, R.: Towards global solutions of optimal discrete-valued control problems. Optim. Control Appl. Methods 33, 576-594 (2012)

[65] Wu, Z.Y., Bai, F.S., Lee, H.W.J., Yang, Y.J.: A filled function method for constrained global optimization. J. Glob. Optim. 39, 495-507 (2007)

[66] Wu, C.Z., Teo, K.L.: Global impulsive optimal control computation. J. Ind. Manag. Optim. 2, 435450 (2006)

[67] Wu, C., Teo, K.L., Li, R., Zhao, Y.: Optimal control of switched systems with time delay. Appl. Math. Lett. 19, 1062-1067 (2006)

[68] Wu, C.Z., Teo, K.L., Rehbock, V.: A filled function method for optimal discrete-valued control problems. J. Glob. Optim. 44, 213-225 (2009)

[69] Xu, X., Antsaklis, P.J.: Optimal control of switched systems based on parameterization of the switching instants. IEEE Trans. Autom. Control 49, 2-16 (2004)

[70] Yu, R., Leung, P.: Optimal partial harvesting schedule for aquaculture operations. Mar. Resour. Econ. 21, 301-315 (2006)

[71] Yu, C., Li, B., Loxton, R., Teo, K.L.: Optimal discrete-valued control computation. J. Glob. Optim. 56, 503-518 (2013)

[72] Yu, C., Teo, K.L., Zhang, L., Bai, Y.: A new exact penalty function method for continuous inequality constrained optimization problems. J. Ind. Manag. Optim. 6, 895-910 (2010)

[73] Yu, C., Teo, K.L., Zhang, L., Bai, Y.: On a refinement of the convergence analysis for the new exact penalty function method for continuous inequality constrained optimization problem. J. Ind. Manag. Optim. 8, 485-491 (2012)

[74] Zhang, L.S., Ng, C.K., Li, D., Tian, W.W.: A new filled function method for global optimization. J. Glob. Optim. 28, 17-43 (2004)

[75] Zhang, L., Wu, S.Y., López, M.A.: A new exchange method for convex semi-infinite programming. SIAM J. Optim. 20, 2959-2977 (2010) 